\title{
SOLITON CELLULAR AUTOMATA ASSOCIATED WITH INFINITE REDUCED WORDS
}

\author{
MAX GLICK, REI INOUE, AND PAVLO PYLYAVSKYY
}

\begin{abstract}
We consider a family of cellular automata $\Phi(n, k)$ associated with infinite reduced elements on the affine symmetric group $\hat{S}_{n}$, which is a tropicalization of the rational maps introduced in GP. We study the soliton solutions for $\Phi(n, k)$ and explore a 'duality' with the $\mathfrak{s l}_{n}$-box-ball system.
\end{abstract}

\section{INTRODUCTION}

1.1. Soliton cellular automata. A soliton cellular automaton (SCA) is a cellular automaton which has solitonic solutions. The first example, and a beautiful one at that, of SCA is the box-ball system (BBS) discovered by Takahashi and Satsuma in 1990 [TS], which is a dynamical system of finitely many balls in an infinite number of boxes arranged in a line. We will present its concrete definition in 97 , and here we simply show a typical time evolution of BBS:

$$
\begin{aligned}
t & =0: \cdots 11222111121111111111111 \cdots \\
t & =1: \cdots 11111222112111111111111 \cdots \\
t & =2: \cdots 11111111221221111111111 \cdots \\
t & =3: \cdots 11111111112112221111111 \cdots \\
t & =4: \cdots 11111111111211112221111 \cdots
\end{aligned}
$$

where 1 and 2 respectively denote an empty box and a box occupied by a ball. One can observe here the notion of soliton that (i) a soliton (a sequence of balls) moves to the right with a constant velocity proportional to the size of the soliton (the length of the sequence), and (ii) a bigger soliton eventually passes a smaller one, after a scattering with resulting 'shifts' of their locations. The shifts caused by scattering provide evidence that the BBS is a nonlinear system.

Though the original definition of BBS seems to be far from known integrable systems, the piecewise-linear equation which describes the system turned out to be related to the piecewise-linear limit (ultradiscretization or tropicalization) of the discrete $\mathrm{KdV}$ equation [TTMS]. Another remarkable property of BBS is that its initial value problem is independently solved by using completely different mathematics, crystal base theory [FOY, HHIKTT] and tropical geometry [IT]. (Also see [IKT] for a review and a list of references on these topics.) In any of these strategies, the tau-function plays an important role in describing solutions [H1].

We are interested in methods to construct SCA and to study their solutions applying combinatorics, representation theory and tropical geometry. To this end, we start with the discrete soliton equations introduced in [GP], and study the corresponding SCA.

Date: December 23, 2017, revised on July 24, 2018.

R. I. was partially supported by JSPS KAKENHI Grant Number 26400037 and 16 H03927.

P. P. was partially supported by NSF grants DMS-1148634, DMS-1351590, and Sloan Fellowship. 
1.2. The Coxeter discrete $\mathbf{K d V}$. In GP, two of the authors introduced a new method to construct dynamical models of discrete space-time coordinates, associated to a pair of reduced words in the affine symmetric group $\hat{S}_{n}$. Let $s_{i}(i=0, \ldots, n-1)$ be the generators of $\hat{S}_{n}$ with relations:

$$
\begin{aligned}
& s_{i}^{2}=1, \\
& s_{i} s_{i+1} s_{i}=s_{i+1} s_{i} s_{i+1}, \\
& s_{i} s_{j}=s_{j} s_{i} \quad|i-j|>1 \quad \bmod n,
\end{aligned}
$$

where we take the indices $i$ of $s_{i}$ modulo $n$. Consider a pair $u, v$ of reduced elements in $\hat{S}_{n}$ such that $v u$ is also reduced. Fix reduced decompositions of $u$ and $v, u=s_{i_{1}} s_{i_{2}} \cdots s_{i_{l}}$ and $v=s_{j_{1}} s_{j_{2}} \cdots s_{j_{m}}$, and assign each $s_{i}$ with a real variable $a$ as $s_{i}(a)$. The dynamics called the 'Coxeter discrete $\mathrm{KdV}$ ' is defined as the rational transformation of the $\ell+m$ variables assigned to $v u$, induced by 'moving $v$ to the right of $u$ ' with the use of the Lusztig relations:

$$
\begin{aligned}
& s_{i}(a) s_{i+1}(b) s_{i}(c)=s_{i+1}(b c /(a+c)) s_{i}(a+c) s_{i+1}(a b /(a+c)), \\
& s_{i}(a) s_{j}(b)=s_{j}(b) s_{i}(a) \quad|i-j|>1 \quad \bmod n .
\end{aligned}
$$

Originally these relations were introduced by Lusztig to study the totally positive parts of algebraic groups and the canonical bases of quantum groups [L, BFZ]. In the network model which offers a strong tool in GP, (1.1) is depicted as in Figure 1 and called the Yang-Baxter move [LP].

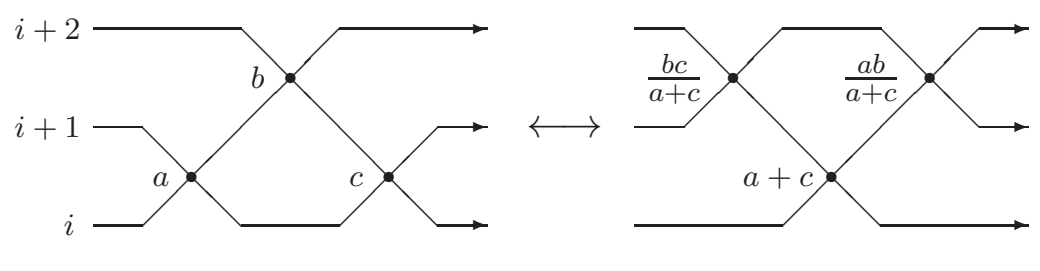

Figure 1. The Yang-Baxter move

The important property of the rational transformation studied in GP is that they have soliton solutions. By applying the network model to a pictorial representation of reduced elements in $\hat{S}_{n}$, we identify vertex variables and chamber variables of the network respectively with the dynamical variables and the tau-functions of the model. This enables us to reduce the rational transformation to a bilinear equation, and the multi-soliton solutions are obtained.

1.3. Main results. In this paper, we focus on a family of dynamical system $\phi(n, k)(k=$ $0,1, \ldots, n-2)$ for $n>2$ given by $u=s_{1} s_{2} \ldots \cdots s_{n-1}$ and $v=s_{k} s_{k-1} \cdots s_{0} s_{n-1} \cdots s_{k+2}$. As in the general case, the rational transformations are subtraction free because they are built out of Lusztig moves (1.1) and hence can be written using only the other three arithmetic operations. Hence $\phi(n, k)$ has a tropicalization (or, piecewise-linear version) obtained by replacing $(+, \times, \div)$ with $(\mathrm{min},+,-)$. We study the tropicalization $\Phi(n, k)$ of $\phi(n, k)$, and mainly consider the soliton solutions for $\Phi(n, k)$ on $\mathbb{Z}$.

Our main results are that a form of each soliton for $\Phi(n, k)$ on $\mathbb{Z}$ is parametrized by $(n-2)$ positive integers (Theorem 2.7), and that the evolution rule of the solitons has a 'duality' with that of a well-known soliton cellular automaton called the $\mathfrak{s l}_{n}$-boxball system [FOY, HHIKTT] (Conjecture 7.4, which is a theorem for $n=3,4$ ). The second result is clarified via the combinatorial $R$-matrix acting on the product of crystals 
corresponding to the symmetric tensor representation of $U_{q}^{\prime}\left(\hat{\mathfrak{s l}}_{n}\right)$. Additionally, we find that the rational map $\phi(n, k)$ is a limit of another $R$-matrix action (Proposition 3.1), namely the geometric $R$-matrix acting on the product of geometric versions of the above (symmetric) crystal and its dual.

We remark that the network model [LP] has a potential to be a useful tool to study integrable rational maps. Once we can formulate the map using a network, we may get not only the information of bilinear equations as in GP, but also the combinatorial information of the Lax form. For an example of the latter application, see [LP].

This paper is organized as follows: in Section 2, following [GP] we define the dynamical system and introduce $\phi(n, k)$ and $\Phi(n, k)$. We give the notion of soliton for $\Phi(n, k)$ in \$2.4, and state the first main result at Theorem 2.7. In Section 3, the explicit formula for $\phi(n, k)$ and the relation to the geometric $R$-matrix is shown (Proposition 3.1). In Section 4, by making use of the pictorial representation of reduced words in $\hat{S}_{n}$, the tau-function and the bilinear equation for the model are obtained. Sections 5 and 6 are devoted to computing soliton solutions for $\Phi(n, k)$ in different two ways. We compute the tropicalization of the geometric solutions for $\phi(n, k)$ obtained in [GP] in Section 5, and see that almost all geometric solutions vanish in tropicalization except for the simplest ones. In Section 6, we naively solve the tropical bilinear equations and prove Theorem 2.7. In Section 7, we study the duality between the soliton solutions for $\Phi(n, k)$ and the $\mathfrak{s l}_{n}$-box-ball system. After a brief introduction of the box-ball system, we present Conjecture 7.4. We explain a strategy to prove it in $\$ 7.3$, and give the proof in the cases of $n=3$ and 4 in $\$ 7.4$ and $\$ 7.5$ respectively. In the last section, we present other interesting numerical phenomena for $\Phi(n, k)$, including negative solitons, pulsars, and relaxations of solitons and pulsars. We add an Appendix to explain the basics of the tropical semifield used in this paper.

\section{Description of the MODEL}

2.1. Discrete dynamical system in infinite reduced words. Following [GP], we introduce a discrete dynamical system associated to a pair of reduced words.

Define an automorphism $\rho$ on the affine symmetric group $\hat{S}_{n}$ by $\rho\left(s_{i}\right)=s_{i+1}$. Let $\eta$ be a homomorphism from $\hat{S}_{n}$ to the symmetric group $S_{n}$ given by $\eta\left(s_{i}\right)=(i, i+1)$ (as usual, $i$ is considered modulo $n$ ). We call a reduced element $g \in \hat{S}_{n}$ a glide, when

$$
\eta(g)=\left(\begin{array}{ccccccc}
1 & 2 & \cdots & n-k & n-k+1 & \cdots & n \\
1+k & 2+k & \cdots & n & 1 & \cdots & k
\end{array}\right)
$$

holds for some $k=0,1, \ldots, n-1$. We call this $k$ the offset of the glides.

Fix the expressions of two glides $u=s_{i_{1}} s_{i_{2}} \cdots s_{i_{l}}$ and $v=s_{j_{1}} s_{j_{2}} \cdots s_{j_{m}}$, and assume that $v u$ is reduced. Let $k_{1}$ and $k_{2}$ be the offsets of $u$ and $v$ respectively. By [GP, Lemma 2.1], we have

$$
v u=\rho^{k_{2}}(u) \rho^{-k_{1}}(v)
$$

Consider an (semi-)infinite word

$$
u \cdot \rho^{-k_{1}}(u) \cdot \rho^{-2 k_{1}}(u) \cdot \rho^{-3 k_{1}}(u) \cdots
$$


Put $v$ on the left side of the infinite word, and move it to the right using (2.1):

$$
\begin{aligned}
v \cdot u & \cdot \rho^{-k_{1}}(u) \cdot \rho^{-2 k_{1}}(u) \cdot \rho^{-3 k_{1}}(u) \cdots \\
& =\rho^{k_{2}}(u) \cdot \rho^{-k_{1}}(v) \cdot \rho^{-k_{1}}(u) \cdot \rho^{-2 k_{1}}(u) \cdot \rho^{-3 k_{1}}(u) \cdots \\
& =\cdots \\
& =\rho^{k_{2}}(u) \cdot \rho^{-k_{1}+k_{2}}(u) \cdot \rho^{-2 k_{1}+k_{2}}(u) \cdot \rho^{-3 k_{1}+k_{2}}(u) \cdots .
\end{aligned}
$$

At the first line of (2.2), we assign positive variables $\mathbf{z}_{0}=\left(z_{0,1}, \ldots, z_{0, m}\right)$ to $v$ as $s_{j_{1}}\left(z_{0,1}\right) \cdots s_{j_{m}}\left(z_{0, m}\right)$, and $\mathbf{y}_{i}=\left(y_{i, 1}, y_{i, 2}, \ldots, y_{i, l}\right)$ to $\rho^{-i k_{1}}(u)$ as $s_{i_{1}-i k_{1}}\left(y_{i, i_{1}}\right) \cdots s_{i_{l}-i k_{1}}\left(y_{i, i_{l}}\right)$ for $i \in \mathbb{Z}_{\geq 0}$. By using the Lusztig relations, we define a rational transformation of the parameters corresponding to (2.2);

$$
\begin{aligned}
\left(\mathbf{z}_{0}, \mathbf{y}_{0}, \mathbf{y}_{1}, \mathbf{y}_{2}, \mathbf{y}_{3}, \ldots\right) & \\
& \mapsto\left(\mathbf{y}_{0}^{\prime}, \mathbf{z}_{1}, \mathbf{y}_{1}, \mathbf{y}_{2}, \mathbf{y}_{3}, \ldots\right) \\
& \mapsto \cdots \\
& \mapsto\left(\mathbf{y}_{0}^{\prime}, \mathbf{y}_{1}^{\prime}, \mathbf{y}_{2}^{\prime}, \mathbf{y}_{3}^{\prime}, \ldots, \mathbf{z}_{\infty}\right)
\end{aligned}
$$

where $\left(\mathbf{z}_{i}, \mathbf{y}_{i}\right)$ is transformed into $\left(\mathbf{y}_{i}^{\prime}, \mathbf{z}_{i+1}\right)$ corresponding to $\rho^{-i k_{1}}(v) \cdot \rho^{-i k_{1}}(u)=\rho^{-i k_{1}+k_{2}}(u)$. $\rho^{-(i+1) k_{1}}(v)$. We call each $\mathbf{y}_{i}$ a state, and each $\mathbf{z}_{i}$ a carrier.

A commuting pair for $u$ and $v$ is a choice of $\mathbf{w}=\left(w_{1}, \ldots, w_{l}\right)$ and $\mathbf{z}=\left(z_{1}, \ldots, z_{m}\right)$ satisfying

$$
\begin{aligned}
& s_{j_{1}}\left(z_{1}\right) s_{j_{2}}\left(z_{2}\right) \cdots s_{j_{m}}\left(z_{m}\right) \cdot s_{i_{1}}\left(w_{1}\right) s_{i_{2}}\left(w_{2}\right) \cdots s_{i_{l}}\left(w_{l}\right) \\
& \quad=s_{i_{1}+k_{2}}\left(w_{1}\right) s_{i_{2}+k_{2}}\left(w_{2}\right) \cdots s_{i_{l}+k_{2}}\left(w_{l}\right) \cdot s_{j_{1}-k_{1}}\left(z_{1}\right) s_{j_{2}-k_{1}}\left(z_{2}\right) \cdots s_{j_{m}-k_{1}}\left(z_{m}\right) .
\end{aligned}
$$

We call such $\mathbf{w}$ and $\mathbf{z}$ a vacuum state and an initial carrier respectively.

We fix a commuting pair $(\mathbf{w}, \mathbf{z})$, we set $\mathbf{z}_{0}=\mathbf{z}$, and we assume $\lim _{i \rightarrow \infty} \mathbf{y}_{i}=\mathbf{w}$. For $t>0$, define $\left(\mathbf{y}_{i}^{t}\right)_{i \in \mathbb{Z}_{\geq 0}}$ inductively by

$$
\left(\mathbf{z}, \mathbf{y}_{0}^{t}, \mathbf{y}_{1}^{t}, \mathbf{y}_{2}^{t}, \mathbf{y}_{3}^{t}, \ldots\right) \mapsto\left(\mathbf{y}_{0}^{t+1}, \mathbf{y}_{1}^{t+1}, \mathbf{y}_{2}^{t+1}, \mathbf{y}_{3}^{t+1}, \ldots\right) .
$$

Empirically, at the end of each step the final carrier $\mathbf{z}_{\infty}$ equals the initial one $\mathbf{z}$ and the new states again satisfy $\lim _{i \rightarrow \infty} \mathbf{y}_{i}^{t+1}=\mathbf{w}$.

Remark 2.1. For the sake of exposition, we have simplified the system from [GP] wherein the state sequence $\ldots, \mathbf{y}_{-1}, \mathbf{y}_{0}, \mathbf{y}_{1}, \mathbf{y}_{2}, \ldots$ is bi-infinite and assumed to approach $\mathbf{w}$ in both directions. The description above corresponds to the special case in which $\mathbf{y}_{i}=\mathbf{w}$ for all $i<0$. To properly define the general system one must insert the initial carrier farther and farther left and take a limit. We ignore this issue, because our focus will be on the tropicalization of the system for which it is consistent to assume for each $t$ that only finitely many $\mathbf{y}_{i}^{t}$ differ from $\mathbf{w}$.

2.2. Dynamical system $\phi(n, k)$. Let us focus on the case that both $u$ and $v$ have length $n-1$ as

$$
\begin{aligned}
& u=s_{1} s_{2} s_{3} \cdots s_{n-1}, \\
& v=v(k)=s_{k} s_{k-1} \cdots s_{0} s_{n-1} \cdots s_{k+2} ; k=0,1, \ldots, n-2,
\end{aligned}
$$

whose offsets are $k_{1}=1$ and $k_{2}=n-1$ respectively.

Lemma 2.2. The following pair $(\mathbf{w}, \mathbf{z})$ is a commuting pair for $(u, v(k))$ :

$$
\begin{aligned}
& \mathbf{z}=\left(\alpha_{k}-\alpha_{k+1}, \alpha_{k-1}-\alpha_{k+1}, \ldots, \alpha_{1}-\alpha_{k+1}, \alpha_{n}-\alpha_{k+1}, \alpha_{n-1}-\alpha_{k+1}, \ldots, \alpha_{k+2}-\alpha_{k+1}\right), \\
& \mathbf{w}=\left(\alpha_{n}-\alpha_{1}, \alpha_{n}-\alpha_{2}, \ldots, \alpha_{n}-\alpha_{n-1}\right),
\end{aligned}
$$


where we assume $\alpha_{k+1}<\alpha_{i}<\alpha_{n}$ for $i=\{1, \ldots, n-1\} \backslash\{k+1\}$, and the indices $i$ of $\alpha_{i}$ are taken modulo $n$.

Proof of Lemma 2.2. To the wiring diagram of $v(k) u$ introduced at Figure 4 in $\$ 4.2$, we apply the wire ansatz in [GP, Section 4] by replacing $\alpha_{i}$ with $-\alpha_{i}$ on the $i$-th wire.

We write $\phi(n, k)$ for the dynamical system (2.4) given by the two glides $u$ and $v(k)$ of (2.5) together with the commuting pair of Lemma 2.2.

Example 2.3. The system $\phi(3,1)$ corresponds to the words $u=s_{1} s_{2}, v=s_{1} s_{0}$. The equation (2.1) reads

$$
v u=s_{1} s_{0} s_{1} s_{2}=s_{0} s_{1} s_{0} s_{2}=\rho^{2}(u) \rho^{-1}(v)
$$

which follows from a single braid move. By (1.1) the weights evolve according to

$$
\begin{aligned}
\left(\left(z_{i, 1}^{t}, z_{i, 2}^{t}\right),\left(y_{i, 1}^{t}, y_{i, 2}^{t}\right)\right) \mapsto & \left(\left(y_{i, 1}^{t+1}, y_{i, 2}^{t+1}\right),\left(z_{i+1,1}^{t}, z_{i+1,2}^{t}\right)\right) \\
= & \left(\left(\frac{z_{i, 2}^{t} y_{i, 1}^{t}}{z_{i, 1}^{t}+y_{i, 1}^{t}}, z_{i, 1}^{t}+y_{i, 1}^{t}\right),\left(\frac{z_{i, 1}^{t} z_{i, 2}^{t}}{z_{i, 1}^{t}+y_{i, 1}^{t}}, y_{i, 2}^{t}\right)\right) .
\end{aligned}
$$

The commuting pair is $\mathbf{z}=\left(\alpha_{1}-\alpha_{2}, \alpha_{3}-\alpha_{2}\right), \mathbf{w}=\left(\alpha_{3}-\alpha_{1}, \alpha_{3}-\alpha_{2}\right)$ and one can check easily from the formula that $(\mathbf{z}, \mathbf{w}) \mapsto(\mathbf{w}, \mathbf{z})$ does hold. The full system inputs $\mathbf{y}_{0}^{t}, \mathbf{y}_{1}^{t}, \ldots$ with $\lim _{i \rightarrow \infty} \mathbf{y}_{i}^{t}=\mathbf{w}$ and uses (2.6) (with $\mathbf{z}_{0}^{t}=\mathbf{z}$ ) to calculate the $\mathbf{y}_{i}^{t+1}$.

2.3. Tropical dynamical system $\Phi(n, k)$. For $A, B, C \in \mathbb{R}$, we define the tropicalization of the Lusztig relations (1.1) and (1.2) as

$$
\begin{aligned}
& s_{i}(A) s_{i+1}(B) s_{i}(C)=s_{i+1}\left(A^{\prime}\right) s_{i}\left(B^{\prime}\right) s_{i+1}\left(C^{\prime}\right), \\
& s_{i}(A) s_{j}(B)=s_{j}(B) s_{i}(A) \quad|i-j|>1 \quad \bmod n,
\end{aligned}
$$

where

$$
\left(A^{\prime}, B^{\prime}, C^{\prime}\right):=(B+C-\min (A, C), \min (A, C), A+B+-\min (A, C)) .
$$

See Appendix A for preliminaries on tropicalization. It is straightforward to tropicalize Lemma 2.2.

Lemma 2.4. The following pair $(\mathbf{W}, \mathbf{Z})$ is a tropical commuting pair for $(u, v(k))$ :

$$
\mathbf{Z}=\left(A_{k}, A_{k-1}, \ldots, A_{1}, A_{n}, A_{n-1}, \ldots, A_{k+2}\right), \quad \mathbf{W}=\left(A_{n}, A_{n}, \ldots, A_{n}\right),
$$

where we assume

$$
A_{k+1}>A_{i}>A_{n} ; i \in\{1, \ldots, n-1\} \backslash\{k+1\} .
$$

Using the tropical Lusztig relations for the glides $u$ and $v(k)$ of (2.5) we define the piecewise-linear transformation of the real variables $\mathbf{Z}_{i}=\left(Z_{i, 1}, \ldots, Z_{i, n-1}\right)$ and $\mathbf{Y}_{i}=$ $\left(Y_{i, 1}, Y_{i, 2}, \ldots, Y_{i, n-1}\right)$ for $i \in \mathbb{Z}_{\geq 0}$ in the same way as (2.3).

We write $\Phi(n, k)$ for the tropical dynamical system given by the two glides (2.5) and the commuting pair (2.9) . As in the rational case, we call each $\mathbf{Z}_{i}$ and each $\mathbf{Y}_{i}$ a carrier and a state respectively. Also, we call $\mathbf{W}$ the vacuum state, and $\mathbf{Z}$ the initial carrier.

Example 2.5. Corresponding to Example 2.3 , we have $\Phi(3,1)$ given by

$$
\begin{aligned}
& \left(\left(Z_{i, 1}^{t}, Z_{i, 2}^{t}\right),\left(Y_{i, 1}^{t}, Y_{i, 2}^{t}\right)\right) \\
& \quad \mapsto\left(\left(Y_{i, 1}^{t+1}, Y_{i, 2}^{t+1}\right),\left(Z_{i+1,1}^{t}, Z_{i+1,2}^{t}\right)\right) \\
& \quad=\left(\left(Z_{i, 2}^{t}+Y_{i, 1}^{t}-\min \left[Z_{i, 1}^{t}, Y_{i, 1}^{t}\right], \min \left[Z_{i, 1}^{t}, Y_{i, 1}^{t}\right]\right),\left(Z_{i, 1}^{t}+Z_{i, 2}^{t}-\min \left[Z_{i, 1}^{t}, Y_{i, 1}^{t}\right], Y_{i, 2}^{t}\right)\right)
\end{aligned}
$$

with the commuting pair $\mathbf{Z}=\left(A_{1}, A_{3}\right)$ and $\mathbf{W}=\left(A_{3}, A_{3}\right)$. 
In this paper, we mainly study the tropical dynamics on $\mathbb{Z} \subset \mathbb{R}$. In particular we consider the case with $A_{n}=0, A_{i}=1$ for $i=\{1, \ldots, n-1\} \backslash\{k+1\}$, and $A_{k+1}>1$, so that the commuting pair is

$$
\mathbf{Z}=(\underbrace{1, \ldots, 1}_{k}, 0, \underbrace{1, \ldots, 1}_{n-2-k}), \quad \mathbf{W}=(0, \ldots, 0) .
$$

2.4. Solitons. We define a one-soliton for $\Phi(n, k)$ to be a finite sequence $\mathbf{X}_{1}, \ldots, \mathbf{X}_{m}$ of non-vacuum states satisfying the following conditions:

(i) The sequence moves to the right with a constant velocity, i.e. for some $a$ and $b$ the input

$$
\mathbf{X}_{1}, \ldots, \mathbf{X}_{M}, \mathbf{W}, \mathbf{W}, \ldots
$$

is carried under $a$ steps of $\Phi(n, k)$ to

$$
\underbrace{\mathbf{W}, \ldots, \mathbf{W}}_{b}, \mathbf{X}_{1}, \ldots, \mathbf{X}_{M}, \mathbf{W}, \mathbf{W}, \ldots
$$

(ii) For each $t$, the final carrier equals the initial one, i.e. $\mathbf{Z}_{i}^{t}=\mathbf{Z}$ for $i \gg 0$ (unlike the rational case, we know of inputs for which this condition fails, see Section 8.2).

An amazing feature of soliton systems is the existence of multi-soliton solutions, which we define in our setting to be an input consisting of several one-solitons separated by vacuums such that

(iii) for $t \gg 0$ the outcome is a collection of one-solitons, arranged in increasing order of velocity from left to right, with the same set of veloicities as the initial solitons.

We will see that different one-solitons can have the same speed and in particular that the components of a multi-soliton for $t \gg 0$ may differ from the initial ones.

For two states $\mathbf{Y}_{i}^{t}$ and $\mathbf{Y}_{i^{\prime}}^{t^{\prime}}$, we say $\mathbf{Y}_{i}^{t}$ is bigger than $\mathbf{Y}_{i^{\prime}}^{t^{\prime}}$, if $Y_{i, j}^{t} \geq Y_{i^{\prime}, j}^{t^{\prime}}$ for all $j=$ $1, \ldots, n-1$ and there is at least one $j$ such that $Y_{i, j}^{t}>Y_{i^{\prime}, j}^{t^{\prime}}$. In the same manner, we say $\mathbf{Y}_{i}^{t}$ is smaller than $\mathbf{Y}_{i^{\prime}}^{t^{\prime}}$, if $Y_{i, j}^{t} \leq Y_{i^{\prime}, j}^{t^{\prime}}$ for all $j=1, \ldots, n-1$ and there is at least one $j$ such that $Y_{i, j}^{t}<Y_{i^{\prime}, j}^{t^{\prime}}$. We say a soliton is positive (resp. negative) when all states of the soliton are bigger (resp. smaller) than the vacuum state.

The following are several examples of positive solitons, where we show $\left(\mathbf{Y}_{i}^{t}\right)_{i}$ for each $t$.

Example 2.6. One-solitons.

(i) $\Phi(3,1)$ :

$$
\begin{aligned}
& t=0:(00)(31)(00)(00)(00)(00)(00)(00)(00) \\
& t=1:(00)(21)(10)(00)(00)(00)(00)(00)(00) \\
& t=2:(00)(11)(20)(00)(00)(00)(00)(00)(00) \\
& t=3:(00)(01)(30)(00)(00)(00)(00)(00)(00) \\
& t=4:(00)(00)(31)(00)(00)(00)(00)(00)(00) \\
& t=5:(00)(00)(21)(10)(00)(00)(00)(00)(00)
\end{aligned}
$$


(ii) $\Phi(4,1)$ :

$$
\begin{aligned}
t & =0:(000)(312)(000)(000)(000)(000)(000) \\
t & =1:(000)(212)(100)(000)(000)(000)(000) \\
t & =2:(000)(112)(200)(000)(000)(000)(000) \\
t & =3:(000)(012)(300)(000)(000)(000)(000) \\
t & =4:(000)(002)(310)(000)(000)(000)(000) \\
t & =5:(000)(001)(311)(000)(000)(000)(000) \\
t & =6:(000)(000)(312)(000)(000)(000)(000) \\
t & =7:(000)(000)(212)(100)(000)(000)(000)
\end{aligned}
$$

For a one-soliton we define its minimal length to be the minimal lattice length the soliton occupies in propagation. We also define the velocity of a soliton, which is the ratio of 'the minimal number of time steps it takes to recover the initial sequence' and 'the lattice length it propagates during the time steps' (in the notation of (i), velocity $=b / a)$. In the first case of Example 2.6, the soliton occupies one lattice at $t=0,4$ and two lattices at $t=1,2,3,5$. The sequence at $t=0$ is recovered at $t=4$, moving one lattice to the right. Hence it has minimal length one, and velocity $1 / 4$. Similarly, the soliton in the second case has minimal length one, and velocity $1 / 6$.

Theorem 2.7. Consider the system $\Phi(n, k)$ on $\mathbb{Z}$ with commuting pair (2.12). Then

$$
X=\left(b_{1}, b_{2}, \ldots, b_{n-1}\right)
$$

is a one-soliton with minimal length one for any $b_{1}, \ldots, b_{n-1} \in \mathbb{Z}_{\geq 1}$ with $b_{k+1}=1$. Its velocity is $\left(\sum_{k=1}^{n-1} b_{k}\right)^{-1}$.

In principle one can verify that $X$ above is a soliton directly using formulas for the system we develop in 93 . Instead we give the proof in 86 at which point we are able to give explicit descriptions of one-solitons in terms of tau-functions (Proposition 6.7). One upshot of this approach is that it mimics what is done for similar systems and suggests that Theorem 2.7 gives all positive solitons (and in particular, all positive solitons have minimal length one). Moreover, the tau-functions could be useful in constructing multisolitons, which we demonstrate in the case of $n=3$.

To denote a positive soliton we use the form at the minimal length of Theorem 2.7, and call it the minimal form of the soliton. For example, in Example 2.6 the one-solitons have the minimal forms $(3,1)$ for $(i)$ and $(3,1,2)$ for (ii). In the rest we often call a positive soliton just 'a soliton'.

Here are examples of soliton scatterings whose combinatorial property will be studied in 87 . 
Example 2.8. Two-solitons.

(i) The case of $\Phi(3,1) ;(1,1) \times(3,1) \mapsto(3,1) \times(1,1)$ :

$$
\begin{aligned}
t & =0:(00)(11)(00)(31)(00)(00)(00)(00)(00)(00)(00)(00) \\
t & =1:(00)(01)(10)(21)(10)(00)(00)(00)(00)(00)(00)(00) \\
t & =2:(00)(00)(11)(11)(20)(00)(00)(00)(00)(00)(00)(00) \\
t & =3:(00)(00)(01)(20)(21)(00)(00)(00)(00)(00)(00)(00) \\
t & =4:(00)(00)(00)(21)(11)(00)(00)(00)(00)(00)(00)(00) \\
t & =5:(00)(00)(00)(11)(20)(11)(00)(00)(00)(00)(00)(00) \\
t & =6:(00)(00)(00)(01)(30)(01)(10)(00)(00)(00)(00)(00) \\
t & =7:(00)(00)(00)(00)(31)(00)(11)(00)(00)(00)(00)(00)
\end{aligned}
$$

(ii) The case of $\Phi(4,1) ;(2,1,1) \times(3,1,2) \mapsto(4,1,1) \times(1,1,2)$ :

$$
\begin{aligned}
t & =0:(000)(211)(001)(311)(000)(000)(000)(000)(000)(000) \\
t & =1:(000)(111)(100)(312)(000)(000)(000)(000)(000)(000) \\
t & =2:(000)(011)(200)(212)(100)(000)(000)(000)(000)(000) \\
t & =3:(000)(001)(210)(112)(200)(000)(000)(000)(000)(000) \\
t & =4:(000)(000)(211)(012)(300)(000)(000)(000)(000)(000) \\
t & =5:(000)(000)(111)(102)(310)(000)(000)(000)(000)(000) \\
t & =6:(000)(000)(011)(201)(311)(000)(000)(000)(000)(000) \\
t & =7:(000)(000)(001)(210)(312)(000)(000)(000)(000)(000) \\
t & =8:(000)(000)(000)(211)(212)(100)(000)(000)(000)(000) \\
t & =9:(000)(000)(001)(111)(302)(110)(000)(000)(000)(000) \\
t & =10:(000)(000)(000)(011)(401)(111)(000)(000)(000)(000) \\
t & =11:(000)(000)(000)(001)(410)(112)(000)(000)(000)(000) \\
t & =12:(000)(000)(000)(000)(411)(012)(100)(000)(000)(000) \\
t & =13:(000)(000)(000)(000)(311)(102)(110)(000)(000)(000) \\
t & =14:(000)(000)(000)(000)(211)(201)(111)(000)(000)(000)
\end{aligned}
$$

where the change of internal structure of solitons is observed.

The behavior witnessed in these and other examples suggest the following.

Conjecture 2.9. Combining positive solitons gives rise to multi-soliton solutions as in condition (iii) at the beginning of this subsection.

In the cases of $n=3,4$, this conjecture is a theorem which follows from the duality with the $\mathfrak{s l}_{n}$-box-ball system proved in $\$ 7.4$ and $\$ 7.5$.

\section{The Formula For $\phi(n, k)$}

Let $\overline{\mathcal{R}}$ be a rational map on $\mathbb{Q}(\mathbf{p}, \mathbf{q})$ with non-negative variables $\mathbf{p}=\left(p_{i}\right)_{i=1, \ldots, n}$, $\mathbf{q}=\left(q_{i}\right)_{i=1, \ldots, n}$, given by $\overline{\mathcal{R}}:(\mathbf{p}, \mathbf{q}) \mapsto\left(\mathbf{q}^{\prime}, \mathbf{p}^{\prime}\right)$;

$$
p_{i}^{\prime}=p_{i} \frac{p_{i+1}+q_{i+1}}{p_{i}+q_{i}}, \quad q_{i}^{\prime}=q_{i} \frac{p_{i+1}+q_{i+1}}{p_{i}+q_{i}} .
$$

This map originates from the geometric version of the combinatorial $R$-matrix, the isomorphism between the tensor products of crystals $B_{\bar{m}} \otimes B_{\ell} \stackrel{\sim}{\rightarrow} B_{\ell} \otimes B_{\bar{m}}$. Here $B_{\ell}$ is the 
crystal corresponding to the $\ell$-fold symmetric tensor representation of $U_{q}^{\prime}\left(\hat{\mathfrak{s l}}_{n}\right)$, and $B_{\bar{\ell}}$ is the dual of $B_{\ell}$. As a set, $B_{\ell}$ and $B_{\bar{\ell}}$ are the same:

$$
B_{\ell}=B_{\bar{\ell}}=\left\{\mathbf{x}=\left(x_{1}, x_{2}, \ldots, x_{n}\right) \in\left(\mathbb{Z}_{\geq 0}\right)^{n} ; \sum_{i=1}^{n} x_{i}=\ell\right\} .
$$

See $[\mathrm{LP}, \S 3.1$ and $\S 11.9]$ for details of the map $\overline{\mathcal{R}}$.

Proposition 3.1. The transformation $\left(\mathbf{z}_{i}, \mathbf{y}_{i}\right) \mapsto\left(\mathbf{y}_{i}^{\prime}, \mathbf{z}_{i+1}\right)$ of the dynamical system $\phi(n, k)$ is described by $\overline{\mathcal{R}}$, by setting

$$
\begin{aligned}
& \mathbf{p}=\left(z_{i, k+1}, z_{i, k+2}, \ldots, z_{i, n-1}, 0, z_{i, 1}, z_{i, 2}, \ldots, z_{i, k}\right), \\
& \mathbf{q}=\left(0, y_{i, n-1}, y_{i, n-2}, \ldots, y_{i, k+2}, y_{i, k+1}, y_{i, k}, \ldots, y_{i, 1}\right) .
\end{aligned}
$$

More explicitly, the transformation $\left(\mathbf{z}_{i}, \mathbf{y}_{i}\right) \mapsto\left(\mathbf{y}_{i}^{\prime}, \mathbf{z}_{i+1}\right)$ is given by

$$
y_{i, j}^{\prime}=y_{i, j} \frac{z_{i, k+2-j}+y_{i, j-1}}{z_{i, k+1-j}+y_{i, j}}, \quad z_{i+1, j}=z_{i, j} \frac{z_{i, j+1}+y_{i, k-j}}{z_{i, j}+y_{i, k+1-j}} .
$$

Here we assume that the second subscript $j$ of $z_{i, j}$ and $y_{i, j}$ is taken modulo $n$, and set $y_{i, n}=z_{i, n}=0$.

First let us prove the following lemma. Assuming $y_{i, 0}=z_{i, 0}=0$, denote

$$
y_{i, j}^{\prime \prime}=y_{i, j} \frac{z_{i, k+2-j}+y_{i, j-1}}{z_{i, k+1-j}+y_{i, j}}, \quad z_{i+1, j}^{\prime \prime}=z_{i, j} \frac{z_{i, j+1}+y_{i, k-j}}{z_{i, j}+y_{i, k+1-j}} .
$$

Lemma 3.2. For any $j$ we have

$$
s_{j}\left(z_{i, k+1-j}\right) s_{j-1}\left(z_{i, k+2-j}+y_{i, j-1}\right) s_{j}\left(y_{i, j}\right)=s_{j-1}\left(y_{i, j}^{\prime \prime}\right) s_{j}\left(z_{i, k+1-j}+y_{i, j}\right) s_{j-1}\left(z_{i+1, k+1-j}^{\prime \prime}\right) .
$$

Proof. Direct substitution:

$$
\frac{\left(z_{i, k+2-j}+y_{i, j-1}\right) y_{i, j}}{z_{i, k+1-j}+y_{i, j}}=y_{i, j}^{\prime \prime}, \quad \frac{z_{i, k+1-j}\left(z_{i, k+2-j}+y_{i, j-1}\right)}{z_{i, k+1-j}+y_{i, j}}=z_{i+1, k+1-j}^{\prime \prime} .
$$

Now we are ready to prove the proposition.

Proof of Proposition 3.1. By the commutativity relations

$$
\begin{aligned}
& s_{k}\left(z_{i, 1}\right) \ldots s_{j}\left(z_{i, k+1-j}\right) \ldots s_{k+2}\left(z_{i, n-1}\right) s_{1}\left(y_{i, 1}\right) \ldots s_{j}\left(y_{i, j}\right) \ldots s_{n-1}\left(y_{i, n-1}\right) \\
& =s_{k}\left(z_{i, 1}\right) \ldots s_{1}\left(z_{i, k}\right) s_{0}\left(z_{i, k+1}\right) s_{1}\left(y_{i, 1}\right) \ldots s_{k}\left(y_{i, k}\right) \\
& \quad \cdot s_{n-1}\left(z_{i, k+2}\right) \ldots s_{k+2}\left(z_{i, n-1}\right) s_{k+1}\left(y_{i, k+1}\right) s_{k+2}\left(y_{i, k+2}\right) \ldots s_{n-1}\left(y_{i, n-1}\right) .
\end{aligned}
$$

Applying the lemma several times we see that

$$
\begin{aligned}
& s_{k}\left(z_{i, 1}\right) \ldots s_{1}\left(z_{i, k}\right) s_{0}\left(z_{i, k+1}\right) s_{1}\left(y_{i, 1}\right) \ldots s_{k}\left(y_{i, k}\right) \\
& =s_{k}\left(z_{i, 1}\right) \ldots s_{1}\left(z_{i, k}\right) s_{0}\left(z_{i, k+1}+y_{i, 0}\right) s_{1}\left(y_{i, 1}\right) \ldots s_{k}\left(y_{i, k}\right) \\
& =s_{0}\left(y_{i, 1}^{\prime \prime}\right) s_{k}\left(z_{i, 1}\right) \ldots s_{2}\left(z_{i, k-1}\right) s_{1}\left(z_{i, k}+y_{i, 1}\right) s_{2}\left(y_{i, 2}\right) \ldots s_{k}\left(y_{i, k}\right) s_{0}\left(z_{i+1, k}^{\prime \prime}\right) \\
& =\ldots=s_{0}\left(y_{i, 1}^{\prime \prime}\right) \ldots s_{k-1}\left(y_{i, k}^{\prime \prime}\right) s_{k}\left(z_{i, 1}+y_{i, k}\right) s_{k-1}\left(z_{i+1,1}^{\prime \prime}\right) \ldots s_{0}\left(z_{i+1, k}^{\prime \prime}\right) \\
& =s_{0}\left(y_{i, 1}^{\prime \prime}\right) \ldots s_{k-1}\left(y_{i, k}^{\prime \prime}\right) s_{k}\left(y_{i, k+1}^{\prime \prime}\right) s_{k-1}\left(z_{i+1,1}^{\prime \prime}\right) \ldots s_{0}\left(z_{i+1, k}^{\prime \prime}\right) .
\end{aligned}
$$


Similarly, by application of the lemma, we get

$$
\begin{aligned}
& s_{n-1}\left(z_{i, k+2}\right) \ldots s_{k+2}\left(z_{i, n-1}\right) s_{k+1}\left(y_{i, k+1}\right) s_{k+2}\left(y_{i, k+2}\right) \ldots s_{n-1}\left(y_{i, n-1}\right) \\
& =s_{n-1}\left(z_{i, k+2}\right) \ldots s_{k+2}\left(z_{i, n-1}\right) s_{k+1}\left(z_{i, 0}+y_{i, k+1}\right) s_{k+2}\left(y_{i, k+2}\right) \ldots s_{n-1}\left(y_{i, n-1}\right) \\
& =\ldots=s_{k+1}\left(y_{i, k+2}^{\prime \prime}\right) \ldots s_{n-2}\left(y_{i, n-1}^{\prime \prime}\right) s_{n-1}\left(z_{i, k+2}+y_{i, n-1}\right) s_{n-2}\left(z_{i+1, k+2}^{\prime \prime}\right) \ldots s_{k+1}\left(z_{i+1, n-1}^{\prime \prime}\right) \\
& =s_{k+1}\left(y_{i, k+2}^{\prime \prime}\right) \ldots s_{n-2}\left(y_{i, n-1}^{\prime \prime}\right) s_{n-1}\left(z_{i+1, k+1}^{\prime \prime}\right) \ldots s_{k+1}\left(z_{i+1, n-1}^{\prime \prime}\right) .
\end{aligned}
$$

Putting the two parts together we get

$$
\begin{aligned}
& s_{0}\left(y_{i, 1}^{\prime \prime}\right) \ldots s_{k-1}\left(y_{i, k}^{\prime \prime}\right) s_{k}\left(y_{i, k+1}^{\prime \prime}\right) s_{k-1}\left(z_{i+1,1}^{\prime \prime}\right) \ldots s_{0}\left(z_{i+1, k}^{\prime \prime}\right) \\
& \quad \cdot s_{k+1}\left(y_{i, k+2}^{\prime \prime}\right) \ldots s_{n-2}\left(y_{i, n-1}^{\prime \prime}\right) s_{n-1}\left(z_{i+1, k+1}^{\prime \prime}\right) \ldots s_{k+1}\left(z_{i+1, n-1}^{\prime \prime}\right) \\
& =s_{0}\left(y_{i, 1}^{\prime \prime}\right) \ldots s_{k-1}\left(y_{i, k}^{\prime \prime}\right) s_{k}\left(y_{i, k+1}^{\prime \prime}\right) s_{k+1}\left(y_{i, k+2}^{\prime \prime}\right) \ldots s_{n-2}\left(y_{i, n-1}^{\prime \prime}\right) \\
& \quad \cdot s_{k-1}\left(z_{i+1,1}^{\prime \prime}\right) \ldots s_{0}\left(z_{i+1, k}^{\prime \prime}\right) s_{n-1}\left(z_{i+1, k+1}^{\prime \prime}\right) \ldots s_{k+1}\left(z_{i+1, n-1}^{\prime \prime}\right) \\
& =s_{0}\left(y_{i, 1}^{\prime \prime}\right) \ldots s_{n-2}\left(y_{i, n-1}^{\prime \prime}\right) s_{k-1}\left(z_{i+1,1}^{\prime \prime}\right) \ldots s_{k+1}\left(z_{i+1, n-1}^{\prime \prime}\right) .
\end{aligned}
$$

This separates into a carrier with parameters $z_{i+1, j}^{\prime \prime}$ and a state with parameters $y_{i, j}^{\prime \prime}$. We conclude that $y_{i, j}^{\prime}=y_{i, j}^{\prime \prime}$ and $z_{i+1, j}=z_{i+1, j}^{\prime \prime}$, as desired.

In the following, we use the same notation $\phi(n, k)$ to denote the map on $\left(\mathbb{R}_{>0}^{n-1}\right)^{2}$ which

comprises the dynamics $\phi(n, k)$, so we write $\phi(n, k):\left(\mathbf{z}_{i}, \mathbf{y}_{i}\right) \mapsto\left(\mathbf{y}_{i}^{\prime}, \mathbf{z}_{i+1}\right)$. Let $\iota$ be a map on $\mathbb{R}^{n-1}$ given by $\mathbf{a}=\left(a_{1}, a_{2}, \ldots, a_{n-1}\right) \mapsto\left(a_{n-1}, a_{n-2}, \ldots, a_{1}\right)$, and let $\tilde{\rho}$ be the map on $\left(\mathbb{R}^{n-1}\right)^{2}$ given by $(\mathbf{a}, \mathbf{b}) \mapsto(\iota(\mathbf{b}), \iota(\mathbf{a}))$.

Proposition 3.3. As maps on $\left(\mathbb{R}_{>0}^{n-1}\right)^{2}$, it holds that

$$
\phi(n, n-2-k)=\tilde{\rho} \circ \phi^{-1}(n, k) \circ \tilde{\rho}
$$

for $k=0,1, \ldots, n-2$.

Proof. For $(\mathbf{z}, \mathbf{y})=\left(z_{j}, y_{j}\right)_{j=1, \ldots, n-1}$ we show $\phi(n, n-2-k) \circ \tilde{\rho} \circ \phi(n, k)(\mathbf{z}, \mathbf{y})=\tilde{\rho}(\mathbf{z}, \mathbf{y})$ by a direct calculation. From (3.3) we write

$$
\left(\mathbf{z}^{\prime}, \mathbf{y}^{\prime}\right):=\phi(n, k)(\mathbf{z}, \mathbf{y})=\left(y_{j} \frac{z_{k+2-j}+y_{j-1}}{z_{k+1-j}+y_{j}}, z_{j} \frac{z_{j+1}+y_{k-j}}{z_{j}+y_{k+1-j}}\right)_{j=1, \ldots, n-1},
$$

where we assume $z_{n}=y_{n}=0$. By substituting this into

$$
\phi(n, n-2-k) \circ \tilde{\rho}\left(\mathbf{z}^{\prime}, \mathbf{y}^{\prime}\right)=\left(y_{n-j}^{\prime} \frac{z_{k+j}^{\prime}+y_{n-j+1}^{\prime}}{z_{k+1+j}^{\prime}+y_{n-j}^{\prime}}, z_{n-j}^{\prime} \frac{z_{n-j-1}^{\prime}+y_{k+2+j}^{\prime}}{z_{n-j}^{\prime}+y_{k+1+j}^{\prime}}\right)_{j=1, \ldots, n-1},
$$

we see the claim.

\section{Chamber variables}

As is typical in integrable systems, we will express various solutions to our systems in terms of tau-functions, by applying Hirota's bilinear method [H1. A tau-function can be though of as an auxiliary collection of variables that have relations among themselves imposed by the original evolution equations. Before proceeding, we define certain networks which provide a good visualization of how the tau-functions fit in. 
4.1. Wiring diagram and chamber variables. Following GP we use the network model introduced in [LP] to describe our system. Consider a semi-infinite cylinder with $n$ horizontal directed wires forming an infinite wiring diagram, which is a pictorial representation of an infinite reduced word in $\hat{S}_{n}$. Away from the crossings, the $n$ wires run along the cylinder at $n$ positions, and corresponding to $s_{i} \in \hat{S}_{n}$ we cross the wires in positions $i$ and $i+1$. Corresponding to the parametrized version $s_{i}(a)$ of $s_{i}$, we assign the crossing with $a$, and call it a vertex variable. The Lusztig relation (1.1) corresponds to the Yang-Baxter move of the wires in positions $i, i+1$ and $i+2$ as in Figure 1.

For $i=1, \ldots, n$, let $e_{i}$ be the $i$-th unit vector in $\mathbb{Z}^{n}$. For integers $1 \leq i, j \leq n$, we set

$$
e_{[i, j]}=\left\{\begin{array}{ll}
\sum_{k=i}^{j} e_{k} & \text { if } i \leq j \\
0 & \text { if } i>j
\end{array} .\right.
$$

For $S \in \mathbb{Z}^{n}$, we often write $S_{i}$ for $S+e_{i}$, and $[S]$ for the class of $S$ in $\mathbb{Z}^{n} / \mathbb{Z} e_{[1, n]}$. Following GP, §5.1] (cf. [BFZ]), we label the chambers of the wiring diagram with elements of $\mathbb{Z}^{n} / \mathbb{Z} e_{[1, n]}$, and at the left end of wires, label the wire at position $i$ with $\alpha_{i} \in \mathbb{R}_{>0}$. Fix a label of one chamber, and extend it to the others by labeling the surrounding four chambers at each crossing of the wires, as shown in Figure 2 .

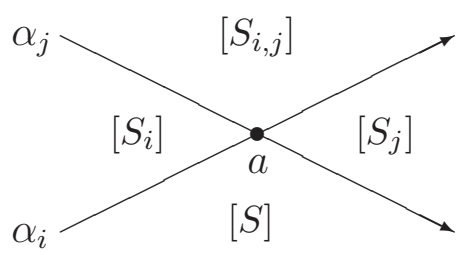

Figure 2. Chamber labeling at the crossing of wires $\alpha_{i}$ and $\alpha_{j}$, where $a$ is the vertex variable associated with this crossing.

The chamber variables make up a tau-function denoted $\tau$ whose domain is $\mathbb{Z}^{n}$. We assume $\tau$ is periodic on a cylinder:

$$
\tau\left(S+e_{[1, n]}\right)=\tau(S) .
$$

The enriched Yang-Baxter move, see Figure 3, corresponds to the relation among the chamber variables

$$
\left(\alpha_{i}-\alpha_{k}\right) \tau\left(S_{j}\right) \tau\left(S_{i, k}\right)=\left(\alpha_{i}-\alpha_{j}\right) \tau\left(S_{k}\right) \tau\left(S_{i, j}\right)+\left(\alpha_{j}-\alpha_{k}\right) \tau\left(S_{i}\right) \tau\left(S_{j, k}\right) .
$$

Remark 4.1. Several names are used in the literature for the enriched Yang-Baxter move: Hirota bilinear difference equation [Z], discrete analogue of generalized Toda equation and lattice KP equation [N], bilinear lattice KP equation [ZFSZ], or Hirota-Miwa equation [LNQ]. It goes back to the works of Miwa [Mi] and Hirota [H1].

The following lemma is shown easily [GP, Lemma 3.1]:

Lemma 4.2. The enriched Yang-Baxter move on chamber variables (4.2) induces the Yang-Baxter move (1.1) on vertex variables via the transformation:

$$
a=\left(\alpha_{i}-\alpha_{j}\right) \frac{\tau\left(S_{i, j}\right) \tau(S)}{\tau\left(S_{i}\right) \tau\left(S_{j}\right)}
$$

with labels as in Figure 0 . 


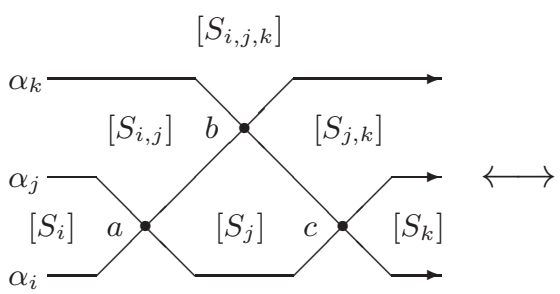

$[S]$

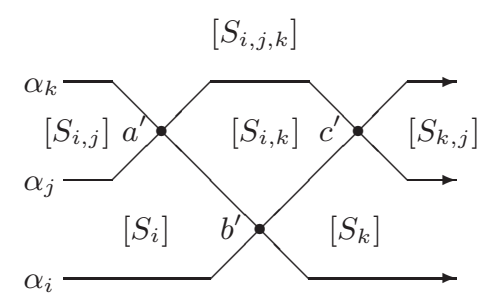

$[S]$

Figure 3. The enriched Yang-Baxter move, where $\left(a^{\prime}, b^{\prime}, c^{\prime}\right)=\left(\frac{b c}{a+c}, a+c, \frac{a b}{a+c}\right)$.

4.2. The tau-function for $\phi(n, k)$. We start with the wiring diagram of the semiinfinite word (2.2) with $u$ and $v=v(k)$ given by (2.5), as depicted at Figure 4 . At the left end of the diagram, the wire at position $i$ is assigned with $\alpha_{i} \in \mathbb{R}_{>0}$, and the chamber between the $n$-th and the 1 -st wires is labelled with $0 \in \mathbb{Z}^{n}$. From the diagram we see that $n-2$ Yang-Baxter moves are applied in calculating the dynamics of $\phi(n, k)$, $\left(\mathbf{z}_{i}^{t}, \mathbf{y}_{i}^{t}\right) \mapsto\left(\mathbf{y}_{i}^{t+1}, \mathbf{z}_{i+1}^{t}\right)$, hence the corresponding enriched Yang-Baxter moves give $n-2$ relations among chamber variables:

$$
\begin{aligned}
& \left(\alpha_{n}-\alpha_{k+1}\right) \tau\left(e_{[1, p-1]}+e_{k+1}\right) \tau\left(e_{[1, p]}-e_{n}\right) \\
& \quad=\left(\alpha_{n}-\alpha_{p}\right) \tau\left(e_{[1, p-1]}+e_{k+1}-e_{n}\right) \tau\left(e_{[1, p]}\right) \\
& \quad+\left(\alpha_{p}-\alpha_{k+1}\right) \tau\left(e_{[1, p-1]}\right) \tau\left(e_{[1, p]}+e_{k+1}-e_{n}\right) ; p=1, \ldots, k \\
& \left(\alpha_{n}-\alpha_{k+1}\right) \tau\left(e_{[1, k+p]}+e_{k+1}\right) \tau\left(e_{[1, k+p+1]}-e_{n}\right) \\
& =\left(\alpha_{n}-\alpha_{k+p+1}\right) \tau\left(e_{[1, k+p]}+e_{k+1}-e_{n}\right) \tau\left(e_{[1, k+p+1]}\right) \\
& \quad+\left(\alpha_{k+p+1}-\alpha_{k+1}\right) \tau\left(e_{[1, k+p]}\right) \tau\left(e_{[1, k+p+1]}+e_{k+1}-e_{n}\right) ; p=1, \ldots, n-k-2,
\end{aligned}
$$

For a glide $g \in \hat{S}_{n}$ we define its trajectory $\kappa(g) \in \mathbb{Z}^{n}$ in the following way: draw the wiring diagram of $g$, and label the wire at position $i$ with $\alpha_{i}$ at the left end. Let $S$ (resp. $S^{\prime}$ ) be the label of the chamber between wires 1 and 2 at the leftmost (resp. rightmost) point of the diagram for $g$. Then the trajectory of $g$ is $\kappa(g):=S^{\prime}-S$.

Lemma 4.3. (i) The trajectories of $v:=v(k)$ and $\tilde{u}:=\rho^{n-1}(u)$ are

$$
\kappa(\tilde{u})=-e_{n}, \quad \kappa(v)=e_{k+1} .
$$

(ii) When we label the chamber to the left of the vertex $y_{0, j}^{t=0}$ with $S \in \mathbb{Z}^{n}$, then that of the vertex $y_{i, j}^{t}$ is

$$
S+i \kappa(\tilde{u})-t \kappa(v) .
$$

Proof. (i) The trajectory $\kappa(v)$ is the difference of the labels of the left chamber of $y_{2}$ and the left chamber of $z_{k}$, which is $\left(e_{1}+e_{k+1}\right)-e_{1}$. Since the offset of $v$ is $k_{2}$, in the diagram the vertex of $y_{1}$ is a crossing of the wires $n$ and 1 . Hence the difference of the labels of the right chamber of $z_{k+1}$ and the left chamber of $y_{1},\left(e_{k+1}-e_{n}\right)-e_{k+1}$, is the trajectory of $\rho^{n-1}(u)$. (ii) It follows from [GP, Proposition 5.4].

Label the left chamber of $z_{i, k+1}^{t}$ with $\kappa(\tilde{u}) i-\kappa(v) t$, and define

$$
\tau^{i, t}(S):=\tau(S+\kappa(\tilde{u}) i-\kappa(v) t)
$$

for $S \in \mathbb{Z}^{n}$. The following proposition immediately follows from Lemma 4.2 . 


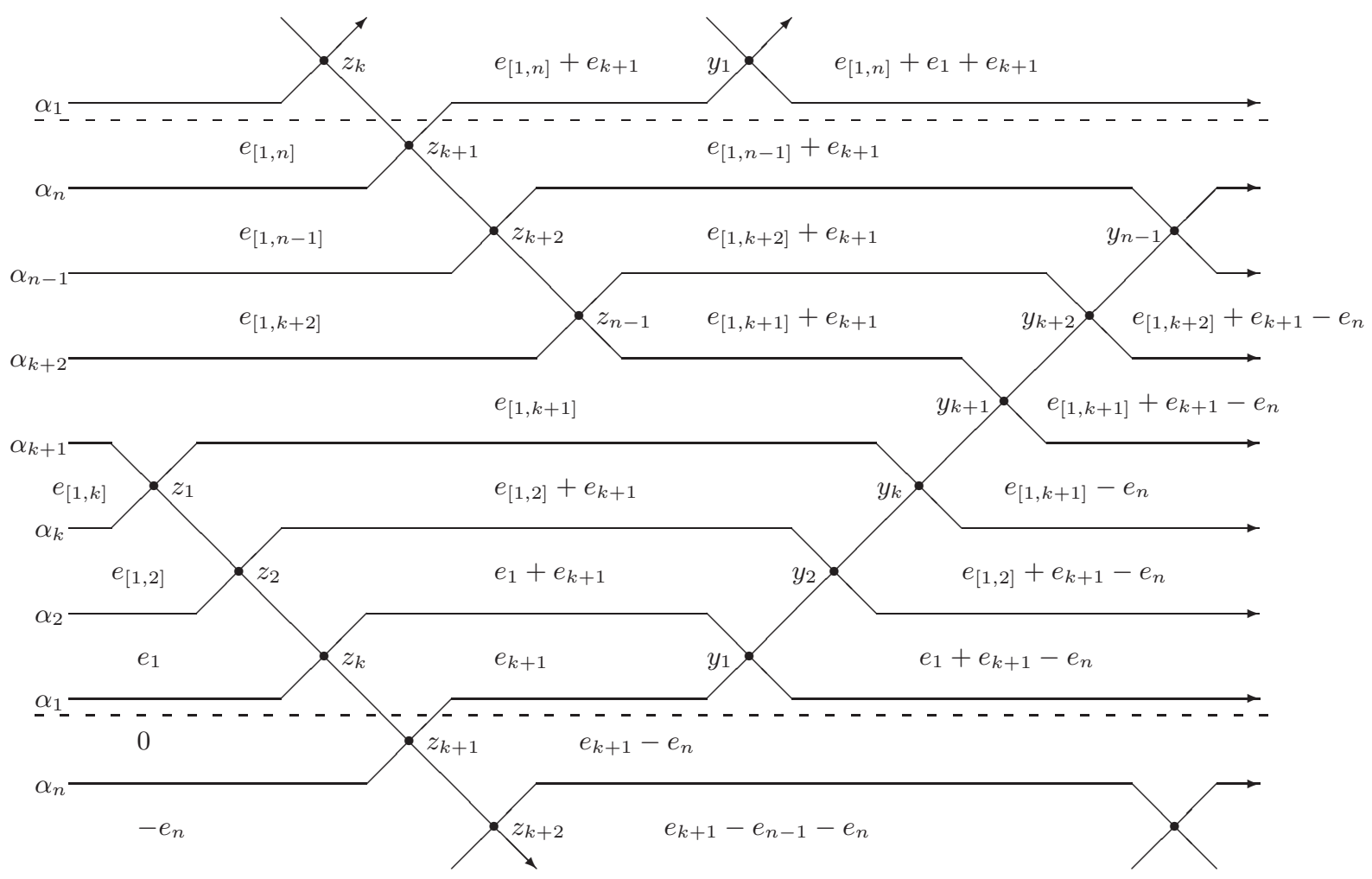

FiguRE 4. The wiring diagram for $v(k) u$ on the universal covering of a cylinder. A fundamental domain is between two dashed lines.

Proposition 4.4. For $i, t \in \mathbb{Z}$, set

$$
\begin{aligned}
& y_{i, p}^{t}=\left(\alpha_{n}-\alpha_{p}\right) \frac{\tau^{i, t}\left(e_{k+1}+e_{[1, p]}\right) \tau^{i, t}\left(e_{k+1}-e_{n}+e_{[1, p-1]}\right)}{\tau^{i, t}\left(e_{k+1}+e_{[1, p-1]}\right) \tau^{i, t}\left(e_{k+1}-e_{n}+e_{[1, p]}\right)} ; p=1, \ldots, n-1, \\
& z_{i, p}^{t}=\left(\alpha_{k+1-p}-\alpha_{k+1}\right) \frac{\tau^{i, t}\left(e_{k+1}+e_{[1, k+1-p]}\right) \tau^{i, t}\left(e_{[1, k-p]}\right)}{\tau^{i, t}\left(e_{[1, k+1-p]}\right) \tau^{i, t}\left(e_{[1, k-p]}+e_{k+1}\right)} ; p=1, \ldots, k, \\
& z_{i, k+p}^{t}=\left(\alpha_{n+1-p}-\alpha_{k+1}\right) \frac{\tau^{i, t}\left(e_{k+1}+e_{[1, n-p+1]}\right) \tau^{i, t}\left(e_{[1, n-p]}\right)}{\tau^{i, t}\left(e_{[1, n-p+1]}\right) \tau^{i, t}\left(e_{k+1}+e_{[1, n-p]}\right)} ; p=1, \ldots, n-k-1 .
\end{aligned}
$$

Then a solution of (4.4) and (4.5) gives the solution for $\Phi(n, k)$.

Via tropicalization, we see the following facts. Let $T$ be the tropical chamber variables (tau-functions) on $\mathbb{Z}^{n}$ satisfying the tropicalization of (4.4) and (4.5):

$$
\begin{aligned}
& T\left(e_{[1, p-1]}+e_{k+1}\right)+T\left(e_{[1, p]}-e_{n}\right) \\
& =\min \left[T\left(e_{[1, p-1]}+e_{k+1}-e_{n}\right)+T\left(e_{[1, p]}\right),\right. \\
& \left.\quad A_{p}-A_{n}+T\left(e_{[1, p-1]}\right)+T\left(e_{[1, p]}+e_{k+1}-e_{n}\right)\right] ; p=1, \ldots, k, \\
& T\left(e_{[1, k+p]}+e_{k+1}\right)+T\left(e_{[1, k+p+1]}-e_{n}\right) \\
& =\min \left[T\left(e_{[1, k+p]}+e_{k+1}-e_{n}\right)+T\left(e_{[1, k+p+1]}\right),\right. \\
& \left.\quad A_{k+p+1}-A_{n}+T\left(e_{[1, k+p]}\right)+T\left(e_{[1, k+p+1]}+e_{k+1}-e_{n}\right)\right] ; p=1, \ldots, n-k-2 .
\end{aligned}
$$


A solution for these equations gives that of $\Phi(n, k)$ via

$$
\begin{aligned}
Y_{i, p}^{t}= & A_{n}+T^{i, t}\left(e_{k+1}+e_{[1, p]}\right)+T^{i, t}\left(e_{k+1}-e_{n}+e_{[1, p-1]}\right) \\
& -T^{i, t}\left(e_{k+1}+e_{[1, p-1]}\right)-T^{i, t}\left(e_{k+1}-e_{n}+e_{[1, p]}\right) ; p=1, \ldots, n-1, \\
Z_{i, p}^{t}= & A_{k+1-p}+T^{i, t}\left(e_{k+1}+e_{[1, k+1-p]}\right)+T^{i, t}\left(e_{[1, k-p]}\right) \\
& -T^{i, t}\left(e_{[1, k+1-p]}\right)-T^{i, t}\left(e_{[1, k-p]}+e_{k+1}\right) ; p=1, \ldots, k \\
Z_{i, k+p}^{t}= & A_{n+1-p}+T^{i, t}\left(e_{k+1}+e_{[1, n-p+1]}\right)+T^{i, t}\left(e_{[1, n-p]}\right) \\
& -T^{i, t}\left(e_{[1, n-p+1]}\right)-T^{i, t}\left(e_{k+1}+e_{[1, n-p]}\right) ; p=1, \ldots, n-k-1 .
\end{aligned}
$$

Here we set $T^{i, t}(S)=T(S+\kappa(\tilde{u}) i-\kappa(v) t)$.

\section{Soliton SOlutions For $\Phi(n, k)$ VIA tropicalization of GeOMEtriC SOLUTION}

We study the soliton solution for $\Phi(n, k)$ on $\mathbb{R}$ by tropicalizing those for $\phi(n, k)$ on $\mathbb{R}_{>0}$ in GP. In this section we prove the following theorem.

Theorem 5.1. Tropicalizing the soliton solutions for $\phi(n, k)$ studied in $\mathrm{GP}$, we get the soliton solutions for $\Phi(n, k)$ whose minimal form is $(x, \ldots, x)$ for $A_{n}<x<A$, where $A=\min \left[A_{i} ; i \in\{1, \ldots, n-1\} \backslash\{k+1\}\right]$. Their velocity is $1 /(n-1)$, independent of $x$.

Besides the condition (2.10), for simplicity we assume that all $A_{i}$ are distinct. Using $\left\{i_{p} ; p=1, \ldots, n\right\}=\{1, \ldots, n\}$ we write the ordering of the $A_{i}$ as

$$
A_{i_{1}}<A_{i_{2}}<\cdots<A_{i_{n}}
$$

where $i_{1}=n$ and $i_{n}=k+1$. We define $A_{\left[i_{1}, \ldots, i_{p}\right]}:=A_{i_{1}}+A_{i_{2}}+\cdots+A_{i_{p}}$. Let $K=\mathbb{C}\{\{t\}\}:=\cup_{n \geq 1} \mathbb{C}\left(\left(t^{1 / n}\right)\right)$ be the field of Puiseux series over $\mathbb{C}$. Let val be the valuation map val $: K \rightarrow \mathbb{R} \cup\{\infty\}$ (see Appendix A.2 for the precise definition.) For $k=1, \ldots, n$, fix $\alpha_{k} \in K$ to satisfy $\operatorname{val}\left(\alpha_{k}\right)=A_{k}$. Let $b, c \in K$ satisfy

$$
\left(b-\alpha_{1}\right)\left(b-\alpha_{2}\right) \cdots\left(b-\alpha_{n}\right)=\left(c-\alpha_{1}\right)\left(c-\alpha_{2}\right) \cdots\left(c-\alpha_{n}\right),
$$

and $A_{i_{p}} \leq \operatorname{val}(b)<\operatorname{val}(c)<A_{i_{p+1}}$ for some $p \in\{1, \ldots, n-1\}$.

Let $F(X, Y)$ be the tropical polynomial:

$$
F(X, Y)=\min \left[Y, n X,(n-1) X+A_{i_{1}},(n-2) X+A_{\left[i_{1}, i_{2}\right]}, \ldots, X+A_{\left[i_{1}, i_{n-1}\right]}, A_{\left[i_{1}, i_{n}\right]}\right] .
$$

The affine tropical curve $\Gamma$ determined by $F(X, Y)$ is a graph in $\mathbb{R}^{2}$ defined as

$$
\Gamma=\left\{(X, Y) \in \mathbb{R}^{2} \mid F(X, Y) \text { is indifferentiable }\right\} .
$$

See Figure 5. The tropical curve $\Gamma$ is the tropicalization of the affine curve $\gamma$ in $K^{2}$ given by

$$
y=y(x)=\left(x-\alpha_{1}\right)\left(x-\alpha_{2}\right) \cdots\left(x-\alpha_{n}\right) .
$$

Precisely, $\Gamma$ is the closure of the set of valuations of points in $\gamma$.

Let $X_{c}$ satisfy $A_{i_{p}}<X_{c}<A_{i_{p+1}}$ for some $p \in\{1,2, \ldots, n-1\}$. We consider a pair of intersection points of the curve $\Gamma$ and a line parallel to the $X$-axis:

$$
\left(A_{i_{p}},(n-p) X_{c}+A_{\left[i_{1}, i_{p}\right]}\right), \quad\left(X_{c},(n-p) X_{c}+A_{\left[i_{1}, i_{p}\right]}\right) .
$$

This pair is the image under val of a pair of points $(b, y(b))$ and $(c, y(c))$ on the curve $\gamma$, such that $y(b)=y(c)$ and $A_{i_{p}} \leq \operatorname{val}(b)<\operatorname{val}(c)=X_{c}<A_{i_{p+1}}$. Note that it turns out that $\operatorname{val}(b)=A_{i_{p}}$ from the graph $\Gamma$. 


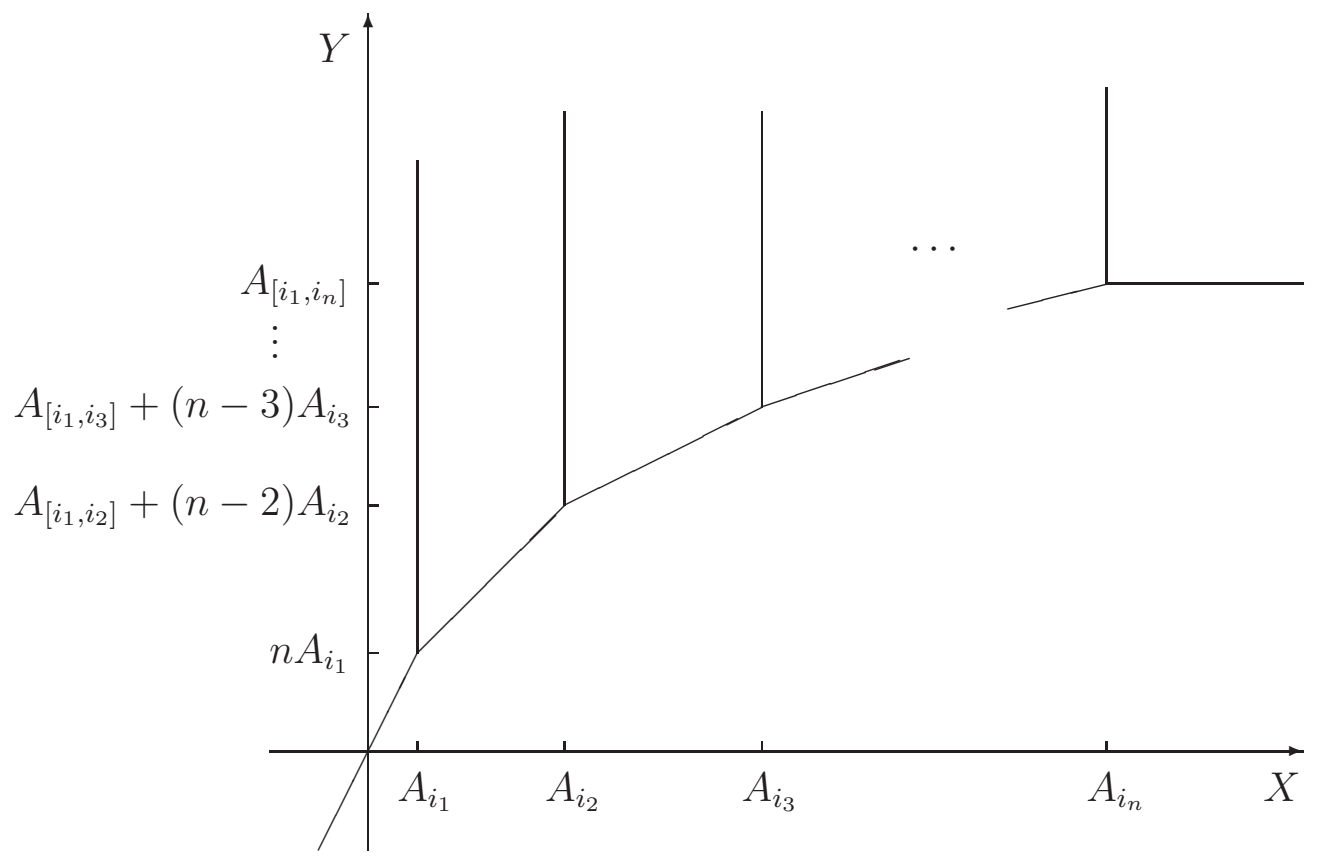

FiguRE 5. The tropical curve $\Gamma$ separating $\mathbb{R}^{2}$ into $n+2$ domains.

Lemma 5.2. It holds that

$$
\begin{aligned}
& \operatorname{val}\left(b+\alpha_{i}\right)= \begin{cases}A_{i} & i=i_{1}, \ldots, i_{p-1}, \\
A_{i_{p}}+(n-p)\left(X_{c}-A_{i_{p}}\right) & i=i_{p}, \\
A_{i_{p}} & i=i_{p+1}, \ldots, i_{n} .\end{cases} \\
& \operatorname{val}\left(c+\alpha_{i}\right)= \begin{cases}A_{i} & i=i_{1}, \ldots, i_{p}, \\
X_{c} & i=i_{p+1}, \ldots, i_{n} .\end{cases}
\end{aligned}
$$

Proof. By the assumption on $X_{c}$ and Lemma A.1, we obtain $\operatorname{val}\left(c-\alpha_{i}\right)$ for all $i$ and $\operatorname{val}\left(b-\alpha_{i}\right)$ for $i \neq i_{p}$ immediately. As for $\operatorname{val}\left(b-\alpha_{i_{p}}\right)$, taking the valuation of (5.1);

$$
\sum_{i=1}^{n} \operatorname{val}\left(b-\alpha_{i}\right)=\sum_{i=1}^{n} \operatorname{val}\left(c-\alpha_{i}\right)
$$

it follows that

$$
\begin{aligned}
\operatorname{val}\left(b-\alpha_{i_{p}}\right) & =\sum_{i=1}^{n} \operatorname{val}\left(c-\alpha_{i}\right)-\sum_{i \neq i_{p}} \operatorname{val}\left(b-\alpha_{i}\right) \\
& =A_{\left[i_{1}, i_{p}\right]}+(n-p) X_{c}-A_{\left[i_{1}, i_{p-1}\right]}-(n-p) A_{i_{p}}
\end{aligned}
$$

and we obtain the result.

The one soliton solution for $\phi(n, k)$ constructed in [GP] is given in terms of chamber variable

$$
\tau(S)=1+\ell B_{1}^{s_{1}} B_{2}^{s_{2}} \cdots B_{n}^{s_{n}} ; \quad S=\left(s_{1}, \ldots, s_{n}\right) \in \mathbb{Z}^{n}, \ell \in K \backslash\{0\}
$$

where

$$
B_{j}=\frac{b-\alpha_{j}}{c-\alpha_{j}} ; j=1, \ldots, n
$$


Due to (5.1), it holds that $\tau\left(S+e_{[1, n]}\right)=\tau(S)$. We are interested in the tropicalization of the tau-function. As a corollary of Lemma 5.2 we obtain the following.

Corollary 5.3. We have

$$
\operatorname{val}\left(B_{i}\right)= \begin{cases}0 & i=i_{1}, \ldots, i_{p-1}, \\ (n-p)\left(X_{c}-A_{i_{p}}\right) & i=i_{p}, \\ A_{i_{p}}-X_{c} & i=i_{p+1}, \ldots, i_{n} .\end{cases}
$$

Remark that $\sum_{i=1}^{n} \operatorname{val}\left(B_{i}\right)=0$ holds. Define $W\left(X_{c}\right):=\left(\operatorname{val}\left(B_{i}\right)\right)_{i=1, \ldots, n} \in \mathbb{R}^{n}$. Now it is easy to show the following.

Proposition 5.4. (i) The one soliton solution for $\Phi(n, k)$ is given by

$$
T(S)=\min \left[0, L+S \cdot W\left(X_{c}\right)\right] ; \quad S \in \mathbb{Z}^{n}, L \in \mathbb{R} .
$$

(ii) We have

$$
\begin{aligned}
& W\left(X_{c}\right) \cdot \kappa\left(\rho^{-1}(u)\right)= \begin{cases}-(n-1)\left(X_{c}-A_{i_{1}}\right) & \text { for } A_{i_{1}}<X_{c}<A_{i_{2}}, \\
0 & \text { for } A_{i_{2}}<X_{c}<A_{i_{n}} .\end{cases} \\
& W\left(X_{c}\right) \cdot \kappa(v)=A_{i_{p}}-X_{c} \text { for } A_{i_{p}}<X_{c}<A_{i_{p+1}} .
\end{aligned}
$$

In particular, the soliton exists only when $A_{i_{1}}<X_{c}<A_{i_{2}}$, and its velocity is $(n-1)^{-1}$, independent of $X_{c}$.

Proof. (i) It follows from Corollary 5.3. (ii) Due to Lemma 4.3 (ii), when $W\left(X_{c}\right)$. $\kappa\left(\rho^{-1}(u)\right) \neq 0$, the velocity of the soliton is given by (Cf. [GP, $\left.\S 8.1\right]$ )

$$
\frac{W\left(X_{c}\right) \cdot \kappa(v)}{W\left(X_{c}\right) \cdot \kappa\left(\rho^{-1}(u)\right)} .
$$

The result is obtained by using Lemma 4.3 (i) and Corollary 5.3 .

By substituting (5.4) into (4.11), we obtain the soliton solution whose minimal form is $(\underbrace{X_{c}, \ldots, X_{c}}_{n-1})$ with $A_{n}<X_{c}<A_{i_{2}}$. Since all of these have velocity $1 /(n-1)$, there is no scattering among solitons. It ends the proof of Theorem 5.1 .

Remark 5.5. For any $N \geq 1$, an $N$-soliton solution corresponding to $X_{n}<X_{c_{1}} \leq X_{c_{2}} \leq$ $\cdots \leq X_{c_{N}}<A$ is given by

$$
T(S)=\min \left[0, \min \left[L_{i}+S \cdot W\left(X_{c_{i}}\right) ; i=1, \ldots, N\right]\right] .
$$

with appropriate $L_{i} \in \mathbb{R}$.

Remark 5.6. When we set $A:=A_{i_{2}}=\cdots=A_{i_{n-1}}$ as (2.12), the tropical curve $\Gamma$ is degenerated and $X_{c}$ has only two possibilities $A_{n}<X_{c}<A$ or $A<X_{c}<A_{k+1}$. The similar calculation of valuation shows

$$
W\left(X_{c}\right)= \begin{cases}\left(X_{c}-A_{n}\right)(-1, \ldots,-1, n-1) & \text { for } A_{n}<X_{c}<A, \\ \frac{X_{c}-A}{n-2}(\underbrace{1, \ldots, 1}_{k},-(n-2), \underbrace{1, \ldots, 1}_{n-k-2}, 0) & \text { for } A<X_{c}<A_{k+1} .\end{cases}
$$

Thus we see that $W\left(X_{c}\right) \cdot \kappa\left(\rho^{-1}(u)\right)$ and $W\left(X_{c}\right) \cdot \kappa(v)$ have the same expression as Proposition 5.4 (ii), and solitons only have volocity $(n-1)^{-1}$. In the limit $X_{c} \rightarrow A$, it gives the positive soliton whose minimal form is $(\underbrace{1,1, \ldots, 1}_{n-1})$ in Theorem 2.7, 


\section{SOLITON SOLUTIONS FOR $\Phi(n, k)$ FROM TROPICAL TAU FUNCTION}

We study the soliton solutions for $\Phi(n, k)$ by naively solving the tropical bilinear equations (4.9) and (4.10), instead of tropicalizing the geometric solutions. It turns out that there are various solitons besides those presented in $\$ 5$, a reflection of the general phenomenon that tropicalization is not reversible. We first present the solution for $\Phi(3, k)$ on $\mathbb{R}$, and next show the solution for $\Phi(n, k)$ on $\mathbb{Z}$ for general $n$. Theorem 2.7 finally follows from Propositions 6.3 and 6.7 .

6.1. The $n=3$ case. Let $\tau(i, t)$ be a function of $(i, t) \in \mathbb{Z}^{2}$ satisfying a relation

$$
\begin{aligned}
& (1+\delta) \tau(i, t-1) \tau(i+2, t+1) \\
& \quad=\tau(i+1, t+1) \tau(i+1, t-1)+\delta \tau(i, t) \tau(i+2, t) .
\end{aligned}
$$

We first demonstrate the case of $\Phi(3,1)$ in detail.

Proposition 6.1. A solution of (6.1) gives the solution of $\phi(3,1)$ via

$$
\begin{aligned}
& y_{i, 1}^{t}=\left(\alpha_{3}-\alpha_{1}\right) \frac{\tau(i+1, t) \tau(i+1, t-1)}{\tau(i, t-1) \tau(i+2, t)}, y_{i, 2}^{t}=\left(\alpha_{3}-\alpha_{2}\right) \frac{\tau(i+1, t-1) \tau(i+2, t)}{\tau(i+1, t) \tau(i+2, t-1)}, \\
& z_{i, 1}^{t}=\left(\alpha_{1}-\alpha_{2}\right) \frac{\tau(i+1, t) \tau(i, t)}{\tau(i+1, t+1) \tau(i, t-1)}, z_{i, 2}^{t}=\left(\alpha_{3}-\alpha_{2}\right) \frac{\tau(i, t-1) \tau(i+1, t)}{\tau(i, t) \tau(i+1, t-1)} .
\end{aligned}
$$

Proof. From (4.4) and (4.5), the bilinear equation for chamber variables $\tau$ of $\phi(3,1)$ is

$$
\begin{aligned}
& \left(\alpha_{3}-\alpha_{2}\right) \tau\left(S+e_{1}-e_{3}\right) \tau\left(S+e_{2}\right) \\
& \quad=\left(\alpha_{3}-\alpha_{1}\right) \tau\left(S+e_{2}-e_{3}\right) \tau\left(S+e_{1}\right)+\left(\alpha_{1}-\alpha_{2}\right) \tau(S) \tau\left(S+e_{1}+e_{2}-e_{3}\right) .
\end{aligned}
$$

We change the chamber coordinate generated by the unit vectors $e_{k}(k=1,2,3)$ to that generated by $\kappa(\tilde{u})=-e_{3}, \kappa(v)=e_{2}$ and $e_{[1,3]}$. By using $e_{1}=e_{[1,3]}+\kappa(\tilde{u})-\kappa(v), e_{2}=\kappa(v)$ and $e_{3}=-t(u)$, and transforming $\tau(i, t, j):=\tau(S)$ when $S=i \cdot \kappa(\tilde{u})-t \cdot \kappa(v)+j \cdot e_{[1,3]}$, we rewrite (6.3) and obtain

$$
\begin{aligned}
& \left(\alpha_{3}-\alpha_{2}\right) \tau(i+2, t+1, j+1) \tau(i, t-1, j) \\
& \quad=\left(\alpha_{3}-\alpha_{1}\right) \tau(i+1, t-1, j+1) \tau(i+1, t+1, j)+\left(\alpha_{1}-\alpha_{2}\right) \tau(i, t, j) \tau(i+2, t, j+1) .
\end{aligned}
$$

We set $\delta=\left(\alpha_{1}-\alpha_{2}\right) /\left(\alpha_{3}-\alpha_{1}\right)$, and ignore the third coordinate by taking into account the periodic condition (4.1). Then (6.1) is obtained.

By identifying $\tau^{i, t}\left(i^{\prime} \cdot \kappa(\tilde{u})-t^{\prime} \cdot \kappa(v)+j^{\prime} \cdot e_{[1,3]}\right)$ with $\tau\left(i+i^{\prime}, t+t^{\prime}, j^{\prime}\right)$ which reduces to $\tau\left(i+i^{\prime}, t+t^{\prime}\right)$, we obtain (6.2) from (4.8).

For $\phi(3,0)$, instead of (6.3) we have

$$
\begin{aligned}
& \left(\alpha_{3}-\alpha_{1}\right) \tau\left(S+e_{[1,2]}-e_{3}\right) \tau\left(S+2 e_{1}\right) \\
& \quad=\left(\alpha_{3}-\alpha_{2}\right) \tau\left(S+2 e_{1}-e_{3}\right) \tau\left(S+e_{[1,2]}\right)+\left(\alpha_{2}-\alpha_{1}\right) \tau\left(S+e_{1}\right) \tau\left(S+e_{[1,2]}+e_{1}-e_{3}\right) .
\end{aligned}
$$

In the same manner using $\kappa(v)=e_{1}$ and $\delta=\left(\alpha_{2}-\alpha_{1}\right) /\left(\alpha_{3}-\alpha_{2}\right)$, we again obtain (6.1). Then a solution of (6.1) gives the solution of $\phi(3,0)$ via

$$
\begin{aligned}
& y_{i, 1}^{t}=\left(\alpha_{3}-\alpha_{1}\right) \frac{\tau(i, t-2) \tau(i+1, t-1)}{\tau(i, t-1) \tau(i+1, t-2)}, y_{i, 2}^{t}=\left(\alpha_{3}-\alpha_{2}\right) \frac{\tau(i+1, t-1) \tau(i+1, t-2)}{\tau(i, t-2) \tau(i+2, t-1)}, \\
& z_{i, 1}^{t}=\left(\alpha_{3}-\alpha_{1}\right) \frac{\tau(i, t-1) \tau(i+1, t)}{\tau(i, t) \tau(i+1, t-1)}, z_{i, 2}^{t}=\left(\alpha_{2}-\alpha_{1}\right) \frac{\tau(i+1, t-1) \tau(i, t-1)}{\tau(i+1, t) \tau(i, t-2)},
\end{aligned}
$$

which originate from (4.8). 
In the following we set $\operatorname{trop}(\delta)=1$, corresponding to the condition (2.12). We study the soliton solutions for $\Phi(3, k)$ by solving the tropicalization of (6.1):

$$
\begin{aligned}
& T(i, t-1)+T(i+2, t+1) \\
& \quad=\min [T(i+1, t+1)+T(i+1, t-1), 1+T(i, t)+T(i+2, t)] .
\end{aligned}
$$

Proposition 6.2. The soliton solutions of (6.5) are given by

(1) one-soliton:

$$
T(i, t)= \begin{cases}\min [0, L+i P-t] & L \in \mathbb{R}, P \geq 2, \\ \min \left[0, L+i P-t \frac{P}{2}\right] & L \in \mathbb{R}, 0<P \leq 2 .\end{cases}
$$

(2) two-soliton: Define $C_{j}(i, t):=L_{j}+i P_{j}-t$ and $C_{j}^{\prime}(i, t):=L_{j}+i P_{j}-t P_{j} / 2$.

$$
T(i, t)=\left\{\begin{array}{c}
\min \left[0, C_{1}(i, t), C_{2}(i, t), C_{1}(i, t)+C_{2}(i, t)+Z_{1,2}\right] ; \\
L_{j} \in \mathbb{R}, P_{j} \geq 2 \text { such that } P_{1} \neq P_{2}, \\
\min \left[0, C_{1}(i, t), C_{2}^{\prime}(i, t), C_{1}(i, t)+C_{2}^{\prime}(i, t)+Z_{1,2}^{\prime}\right] \\
L_{j} \in \mathbb{R}, 0<P_{2}<2 \leq P_{1}
\end{array}\right.
$$

where

$$
Z_{1,2}:=2 \min \left[P_{1}, P_{2}\right]-1, \quad Z_{1,2}^{\prime}:=\frac{3}{2} P_{2} .
$$

Proof. We compute the soliton solutions using Hirota's method [H1, §1.5]. (1) A one soliton solution for (6.1) is set to be a form as $\tau(i, t)=1+\ell p^{i} q^{t}$ with $\ell, p, q \in \mathbb{R}_{>0}$. By substituting it into (6.1), we obtain an algebraic equation for $p$ and $q$ :

$$
(1+\delta)\left(q^{-1}+p^{2} q\right)=p q+p q^{-1}+\delta\left(1+p^{2}\right) .
$$

Then $P:=\operatorname{trop}(p)$ and $Q:=\operatorname{trop}(q)$ are required to satisfy

$$
\min [-Q, 2 P+Q]=\min [P+Q, P-Q, 1+\min [0,2 P]] .
$$

We solve this equation assuming $P>0$ without loss of generality. When $Q>0$, (6.7) reduces to $-Q=\min [P-Q, 1]$, which has no solution. When $Q \leq 0$, (6.7) reduces to $\min [-Q, 2 P+Q]=\min [P+Q, 1]$. If $-Q \leq 2 P+Q$ and $1 \leq P+Q$, we have $Q=-1$ and $P \geq 2$. If $-Q \leq 2 P+Q$ and $1>P+Q$, we have $Q=-P / 2$ and $0<P<2$. If $-Q>2 P-Q$, we have no solution.

(2) By substituting a form of two soliton solution $\tau(i, t)=1+\ell_{1} p_{1}^{i} q_{1}^{t}+\ell_{2} p_{2}^{i} q_{2}^{t}+$ $z_{1,2} \ell_{1} p_{1}^{i} q_{1}^{t} \ell_{2} p_{2}^{i} q_{2}^{t}$ with $\ell_{i}, p_{i}, q_{i}>0$ for $i=1,2$ into (6.1), and taking the order of $\ell_{1} \ell_{2}$, we have

$$
z_{1,2}=\frac{p_{1} p_{2}\left(q_{1}^{2}+q_{2}^{2}\right)+\delta q_{1} q_{2}\left(p_{1}^{2}+p_{2}^{2}\right)-(1+\delta)\left(p_{1}^{2} q_{1}^{2}+p_{2}^{2} q_{2}^{2}\right)}{(1+\delta)\left(1+p_{1}^{2} q_{1}^{2} p_{2}^{2} q_{2}^{2}\right)-\left(p_{1} p_{2}\left(1+\delta p_{1} p_{2} q_{1} q_{2}\right)+q_{1} q_{2}\left(\delta+p_{1} p_{2} q_{1} q_{2}\right)\right)} .
$$

Set $\operatorname{trop}\left(q_{i}\right)=Q_{i}=-1$ and $\operatorname{trop}\left(p_{i}\right)=P_{i} \geq 2$ for $i=1,2$ such that $P_{1} \neq P_{2}$. In the numerator of $z_{1,2}$, we have

$\operatorname{trop}\left(p_{1} p_{2}\left(q_{1}^{2}+q_{2}^{2}\right)+\delta q_{1} q_{2}\left(p_{1}^{2}+p_{2}^{2}\right)\right)=\min \left[P_{1}+P_{2}-2,2 P_{1}-1,2 P_{2}-1\right]=2 \min \left[P_{1}, P_{2}\right]-1$, $\operatorname{trop}\left((1+\delta)\left(p_{1}^{2} q_{1}^{2}+p_{2}^{2} q_{2}^{2}\right)\right)=2 \min \left[P_{1}, P_{2}\right]-2$,

and in the denominator, we have

$$
\begin{aligned}
& \operatorname{trop}\left((1+\delta)\left(1+p_{1}^{2} q_{1}^{2} p_{2}^{2} q_{2}^{2}\right)\right)=\min \left[0,2\left(P_{1}+P_{2}\right)-4\right]=0, \\
& \operatorname{trop}\left(p_{1} p_{2}\left(1+\delta p_{1} p_{2} q_{1} q_{2}\right)+q_{1} q_{2}\left(\delta+p_{1} p_{2} q_{1} q_{2}\right)\right)=\min \left[P_{1}+P_{2},-1, P_{1}+P_{2}-4\right]=-1 .
\end{aligned}
$$


Thus the dominant terms of tropicalization in the numerator and denominator of $z_{i, 2}$ have the same sign, and we obtain

$$
Z_{1,2}:=\operatorname{trop}\left(z_{1,2}\right)=2 \min \left[P_{1}, P_{2}\right]-2-(-1)=2 \min \left[P_{1}, P_{2}\right]-1 .
$$

In the same manner, when $Q_{1}=-1, P_{1} \geq 2, Q_{2}=-P_{2} / 2$ and $0<P_{2}<2$, we obtain $\operatorname{trop}\left(z_{1,2}\right)=3 / 2 P_{2}$.

By substituting (6.6) into the tropicalization of (6.2) or (6.4) we obtain the following:

Proposition 6.3. Assume $L \in \mathbb{Z}$ and $P \in \mathbb{Z}_{>2}$ in (6.6). Then we obtain a positive soliton whose minimal form is $(P-1,1)$ for $\Phi(3,1)$, and a positive soliton whose minimal form is $(1, P-1)$ for $\Phi(3,0)$. In both cases the velocity of soliton is $1 / P$.

The proof is included in that of Proposition 6.7 (ii).

Remark 6.4. For general $L \in \mathbb{R}$ and $P \in \mathbb{R}_{>0}$, a soliton may have the minimal length more than one. See $\oint 8.3$.

6.2. General $n$ case. In the same way as the $n=3$ case, we study soliton solutions for $\Phi(n, k)$ for $n>3$. Unfortunately we obtain only one-soliton solutions for technical reason. For simplicity we study only integral solutions.

We transform the bilinear equations for $\tau^{i, t}$ on the chambers, (4.4) and (4.5), to those for an $(n-2)$-tuple of tau-functions $\left(\tau_{p}\right)_{p=0,1, \ldots, n-3}$ on $\mathbb{Z}^{2}$ using the following rule. Recall the definition of $\tau^{i, t}$ (4.7) and that we have labelled the left chamber of $z_{i, k+1}^{t}$ with $\kappa(\tilde{u}) i-\kappa(v) t$. Note that in the universal covering of the wiring diagram for $v(k) u$, the chamber labels lie in the subset of $\mathbb{Z}^{n}$

$$
\mathcal{C}_{k}:=\left\{e_{[1, p]}+i \cdot e_{n}+t \cdot e_{k+1}+j \cdot e_{[1, n]} ; p \in\{0,1, \ldots, n-1\}, i, t, j \in \mathbb{Z}\right\} .
$$

When $k \geq 1$, we set

$$
\tau^{i, t}\left(e_{[1, p]}\right)= \begin{cases}\tau_{0}(i, t) & \text { if } p=0, \\ \tau_{p}(i+1, t+1) & \text { if } p=1, \ldots, k, \\ \tau_{p-1}(i+1, t) & \text { if } p=k+1, \ldots, n-2, \\ \tau_{0}(i+1, t) & \text { if } p=n-1,\end{cases}
$$

and uniquely extend it to $\mathcal{C}_{k}$ using

$$
\tau^{i, t}\left(-i^{\prime} e_{n}-t^{\prime} e_{k+1}+j e_{[1, n]}\right)=\tau^{i+i^{\prime}, t+t^{\prime}}(0)
$$

for any $i^{\prime}, t^{\prime}, j \in \mathbb{Z}$. For instance, when $n=4$ and $k=2$, it holds that $\tau^{i, t}\left(e_{k+1}+e_{[1,2]}\right)=$ $\tau^{i, t-1}\left(e_{[1,2]}\right)=\tau_{2}(i+1, t)$. Remark that the periodic condition for chamber variables (4.1) is hidden by (6.9). When $k=0$, instead of (6.8) we set

$$
\tau^{i, t}\left(e_{[1, p]}\right)= \begin{cases}\tau_{0}(i, t) & \text { if } p=0 \\ \tau_{0}(i, t-1) & \text { if } p=1 \\ \tau_{p-1}(i+1, t) & \text { if } p=2, \ldots, n-2, \\ \tau_{0}(i+1, t) & \text { if } p=n-1,\end{cases}
$$

and use (6.9).

Proposition 6.5. Set $\alpha_{i}=\alpha$ for $i=\{1,2, \ldots, n-1\} \backslash\{k+1\}$, and define $\delta:=$ $\left(\alpha-\alpha_{k+1}\right) /\left(\alpha_{n}-\alpha\right)$. Via the above introduced transformation, (4.4) and (4.5) reduce to 
the following equations for the $\tau_{p}(i, t)$, which are 'independent' of $k$ :

$$
\begin{aligned}
& (1+\delta) \tau_{0}(i, t-1) \tau_{1}(i+2, t+1) \\
& \quad=\tau_{1}(i+1, t+1) \tau_{0}(i+1, t-1)+\delta \tau_{0}(i, t) \tau_{1}(i+2, t) \\
& (1+\delta) \tau_{p}(i+1, t-1) \tau_{p+1}(i+2, t) \\
& \quad=\tau_{p+1}(i+1, t) \tau_{p}(i+2, t-1)+\delta \tau_{p}(i+1, t) \tau_{p+1}(i+2, t-1) ; p=1, \ldots, n-3,
\end{aligned}
$$

where we assume $\tau_{n-2}(i, t)=\tau_{0}(i, t)$.

Proof. When $k \geq 1$, from (4.4) and (4.5) we obtain

$$
\begin{aligned}
& \left(\alpha_{n}-\alpha_{k+1}\right) \tau_{0}(i, t-1) \tau_{1}(i+2, t+1) \\
& \quad=\left(\alpha_{n}-\alpha_{1}\right) \tau_{1}(i+1, t+1) \tau_{0}(i+1, t-1)+\left(\alpha_{1}-\alpha_{k+1}\right) \tau_{0}(i, t) \tau_{1}(i+2, t), \\
& \left(\alpha_{n}-\alpha_{k+1}\right) \tau_{p-1}(i+1, t) \tau_{p+1}(i+2, t+1) \\
& =\left(\alpha_{n}-\alpha_{p}\right) \tau_{p}(i+1, t+1) \tau_{p-1}(i+2, t)+\left(\alpha_{p}-\alpha_{2}\right) \tau_{p-1}(i+1, t+1) \tau_{p}(i+2, t) ; \\
& \quad p=2, \ldots, k \\
& \left(\alpha_{n}-\alpha_{k+1}\right) \tau_{p-2}(i+1, t-1) \tau_{p-1}(i+2, t) \\
& \quad\left(\alpha_{n}-\alpha_{p}\right) \tau_{p-1}(i+1, t) \tau_{p-2}(i+2, t-1)+\left(\alpha_{p}-\alpha_{k+1}\right) \tau_{p-2}(i+1, t) \tau_{p-1}(i+2, t-1) ; \\
& \quad p=k+2, \ldots, n-1 .
\end{aligned}
$$

Using the defined $\delta$, we see that the first equation reduces to (6.10), and the next two equations reduce to (6.11).

When $k=0$, we only have (4.5) which turns out to be (6.10) when $p=1$, and (6.11) when $p=2,3, \ldots, n-2$.

From Proposition 4.4 we obtain the following:

Corollary 6.6. A solution of (6.10) and (6.11) gives the solution of $\phi(n, k)$ via the following formulae. In the case of $k \geq 1$ :

$$
\begin{aligned}
& y_{i, 1}^{t}=\left(\alpha_{n}-\alpha\right) \frac{\tau_{1}(i+1, t) \tau_{0}(i+1, t-1)}{\tau_{0}(i, t-1) \tau_{1}(i+2, t)} \\
& y_{i, p}^{t}=\left(\alpha_{n}-\alpha\right) \frac{\tau_{p}(i+1, t) \tau_{p-1}(i+2, t)}{\tau_{p-1}(i+1, t) \tau_{p}(i+2, t)} ; p=2, \ldots, k \\
& y_{i, k+1}^{t}=\left(\alpha_{n}-\alpha\right) \frac{\tau_{k}(i+1, t-1) \tau_{k}(i+2, t)}{\tau_{k}(i+1, t) \tau_{k}(i+2, t-1)}, \\
& y_{i, p}^{t}=\left(\alpha_{n}-\alpha\right) \frac{\tau_{p-1}(i+1, t-1) \tau_{p-2}(i+2, t-1)}{\tau_{p-2}(i+1, t-1) \tau_{p-1}(i+2, t-1)} ; p=k+2, \ldots, n-1, \\
& z_{i, p}^{t}=\left(\alpha-\alpha_{k+1}\right) \frac{\tau_{k+1-p}(i+1, t) \tau_{k-p}(i+1, t+1)}{\tau_{k+1-p}(i+1, t+1) \tau_{k-p}(i+1, t)} ; p=1, \ldots, k-1, \\
& z_{i, k}^{t}=\left(\alpha-\alpha_{k+1}\right) \frac{\tau_{1}(i+1, t) \tau_{0}(i, t)}{\tau_{1}(i+1, t+1) \tau_{0}(i, t-1)}, \\
& z_{i, k+1}^{t}=\left(\alpha_{n}-\alpha_{k+1}\right) \frac{\tau_{0}(i, t-1) \tau_{0}(i+1, t)}{\tau_{0}(i, t) \tau_{0}(i+1, t-1)} . \\
& z_{i, k+p}^{t}=\left(\alpha-\alpha_{k+1}\right) \frac{\tau_{n-p}(i+1, t-1) \tau_{n-p-1}(i+1, t)}{\tau_{n-p}(i+1, t) \tau_{n-p-1}(i+1, t-1)} ; p=2, \ldots, n-k-1 .
\end{aligned}
$$


In the case of $k=0$ :

$$
\begin{aligned}
& y_{i, 1}^{t}=\left(\alpha_{n}-\alpha\right) \frac{\tau_{0}(i, t-2) \tau_{0}(i+1, t-1)}{\tau_{0}(i, t-1) \tau_{0}(i+1, t-2)}, \\
& y_{i, 2}^{t}=\left(\alpha_{n}-\alpha\right) \frac{\tau_{1}(i+1, t-1) \tau_{0}(i+1, t-2)}{\tau_{0}(i, t-2) \tau_{1}(i+2, t-1)},
\end{aligned}
$$

and $y_{i, p}^{t}$ for $p=3, \ldots, n-1$ has the same expression as (6.15). The variable $z_{i, 1}^{t}$ is as (6.18), $z_{i, p}^{t}$ for $p=2, \ldots, n-2$ is as (6.19). The variable $z_{i, n-1}^{t+1}$ has the same expression as (6.17).

In the following, we set $\operatorname{trop}(\delta)=1$ corresponding to (2.12), and study solutions of the tropicalization of (6.10) and (6.11):

$$
\begin{aligned}
& T_{0}(i, t-1)+T_{1}(i+2, t+1) \\
& \quad=\min \left[T_{1}(i+1, t+1)+T_{0}(i+1, t-1), 1+T_{0}(i, t)+T_{1}(i+2, t)\right], \\
& T_{p}(i+1, t-1)+T_{p+1}(i+2, t) \\
& =\min \left[T_{p+1}(i+1, t)+T_{p}(i+2, t-1), 1+T_{p}(i+1, t)+T_{p+1}(i+2, t-1)\right], \\
& \quad p=1, \ldots, n-3 .
\end{aligned}
$$

Proposition 6.7. (i) A one-soliton solution for $\Phi(n, k)$ is given by

$$
\begin{aligned}
& T_{0}(i, t)=\min [0, L+i P-t], \\
& T_{p}(i, t)=\min \left[0, L+i P-t+\sum_{j=p}^{n-3} R_{j}\right] ; \quad p=1, \ldots, n-3,
\end{aligned}
$$

where $L \in \mathbb{Z}, P \in \mathbb{Z}_{\geq n-1}, R_{j} \in \mathbb{Z}_{\leq-1}$ for $j=1, \ldots, n-3$, and $P+\sum_{j=1}^{n-3} R_{j} \geq 2$.

(ii) The minimal form of this soliton is

$$
\left(P-1+\sum_{j=1}^{n-3} R_{j},-R_{1},-R_{2}, \ldots,-R_{k-1}, 1,-R_{k}, \ldots,-R_{n-3}\right) .
$$

Proof. (i) We substitute $\tau_{0}(i, t)=1+\ell c^{i} q^{t}$ and $\tau_{s}(i, t)=1+\ell c^{i} q^{t} \prod_{k=s}^{n-3} r_{k}$ into (6.10) and (6.11), and obtain

$$
\begin{aligned}
& (1+\delta)\left(c^{-1}+c^{2} q r\right)=c q r+c q^{-1}+\delta\left(1+c^{2} r\right), \\
& (1+\delta)\left(q^{-1} r_{s}+c\right)=1+c q^{-1} r_{s}+\delta\left(r_{s}+c q^{-1}\right) ; \quad s=1, \ldots, n-3,
\end{aligned}
$$

where $r:=\prod_{k=1}^{n-3} r_{k}$. By eliminating the $r_{s}$, we have

$$
(1+\delta-c-\delta q)^{n-2}=c q(q+\delta c-(1+\delta) c q)^{n-2} .
$$

Assume $P:=\operatorname{trop}(c)>0$ and set $Q:=\operatorname{trop}(q)$ as in the $n=3$ case.

When $n$ is odd, set $n_{0}:=\frac{n-3}{2}$. Eq. (6.24) is expanded as

$$
\begin{aligned}
& \sum_{i=0}^{n_{0}}\left(\begin{array}{c}
n-2 \\
2 i
\end{array}\right)(1+\delta)^{n-2-2 i}(c+\delta q)^{2 i}+c q \sum_{i=0}^{n_{0}}\left(\begin{array}{c}
n-2 \\
2 i+1
\end{array}\right)(q+\delta c)^{n-3-2 i}(c q(1+\delta))^{2 i+1} \\
& =\sum_{i=0}^{n_{0}}\left(\begin{array}{c}
n-2 \\
2 i+1
\end{array}\right)(1+\delta)^{n-3-2 i}(c+\delta q)^{2 i+1}+p q \sum_{i=0}^{n_{0}}\left(\begin{array}{c}
n-2 \\
2 i
\end{array}\right)(q+\delta c)^{n-2-2 i}(c q(1+\delta))^{2 i} .
\end{aligned}
$$


Assume $Q \in \mathbb{Z}_{<0}$. It holds that $\operatorname{trop}(c+\delta q)=\min [P, Q+1]=Q+1 \leq 0$ and $\operatorname{trop}(q+\delta c)=\min [Q, 1+P]=Q$. Thus the tropicalization of (6.25) reduces to

$$
\min [(n-3)(Q+1), 2 P+2 Q+(n-3) Q]=\min [(n-2)(Q+1), P+Q+(n-2) Q],
$$

from which we obtain $\min [n-3,2 P+2 Q]=Q+\min [P+Q, n-2]$. When $P+Q \geq n-2$, it follows that $Q=-1$ and $P \geq n-1$. When $n_{0}<P+Q<n-2$, it holds that $n-3=P+2 Q$ which contradicts $Q \in \mathbb{Z}_{<0}$. When $P+Q \leq n_{0}$, we obtain $P=0$ which is a contradiction. When $Q \geq 0$, there is no solution since the tropicalization of the l.h.s. and the r.h.s of (6.25) are respectively zero and positive.

When $n$ is even, by a similar discussion we see that the solution is that $Q=-1$ and $P \in \mathbb{Z}_{\geq n-1}$.

From (6.23) with $Q=-1$ and $P \geq n-1$, we obtain $\min \left[R_{s}+1, P\right]=\min [0, P+1+$ $\left.R_{s}, 1+R_{s}, 2+P\right]$, which holds for any $R_{s} \leq-1$. Further, from (6.22) it follows that $P+\sum_{k=1}^{n-3} R_{k} \geq 2$.

(ii) Define $\Delta_{p}:=\sum_{i=p}^{n-3} R_{i}$ for $p=1, \ldots, n-3$. We show that the minimal form is obtained by setting $t=1, i=-1$ and $L=-\Delta_{k}$, for $k \geq 2$. The case of $k=1$ is similar.

First, we consider $Y_{i, k+1}^{t=1}$ with $L=-\Delta_{k}$. By tropicalizing (6.14) we have

$$
\begin{aligned}
Y_{i, k+1}^{t=1} & =T_{k}(i+1,0)+T_{k}(i+2,1)-T_{k}(i+1,1)-T_{k}(i+2,0) \\
& =\min [0,(i+1) P]+\min [0,(i+2) P-1]-\min [0,(i+1) P-1]-\min [0,(i+2) P] .
\end{aligned}
$$

When $i=-1$, all but the third term are zero in the last line of the above formula, and we obtain $Y_{-1, k+1}^{1}=1$. It is satisfied that $\min [0, *]=*$ for all terms when $i<-1$, and that $\min [0, *]=0$ for all terms when $i>-1$. Thus $Y_{i, k+1}^{t=1}=0$ when $i \neq-1$.

Next we calculate $Y_{i, 1}^{1}$. By tropicalizing (6.12) we have

$$
\begin{aligned}
Y_{i, 1}^{1}= & T_{1}(i+1,1)+T_{0}(i+1,0)-T_{0}(i, 0)-T_{1}(i+2,1) \\
= & \min \left[0, L+(i+1) P-1+\Delta_{1}\right]+\min [0, L+(i+1) P] \\
& \quad-\min [0, L+i P]-\min \left[0, L+(i+2) P-1+\Delta_{1}\right] .
\end{aligned}
$$

When $i=-1$, the first and the third terms in the above formula are nonzero, and we obtain $Y_{-1,1}^{1}=\left(L-1+\Delta_{1}\right)-(L-P)=P+\Delta_{1}-1$. When $i \neq-1$, we obtain $Y_{i, 1}^{1}=0$ for the same reason as for $Y_{i, k+1}^{1}$.

In the case of $Y_{i, k}^{1}$, from (6.13) we obtain

$$
\begin{aligned}
Y_{i, k}^{1}= & T_{k}(i+1,1)+T_{k-1}(i+2,1)-T_{k-1}(i+1,1)-T_{k}(i+2,1) \\
= & \min [0,(i+1) P-1]+\min \left[0, L+(i+2) P-1+\Delta_{k-1}\right] \\
& \quad-\min \left[0, L+(i+1) P-1+\Delta_{k-1}\right]-\min [0,(i+2) P-1] .
\end{aligned}
$$

Using the conditions $L+\Delta_{k-1}=R_{k-1}, R_{j} \leq-1$ and $P+\sum_{k=1}^{n-3} R_{k} \geq 2$, we see that $Y_{i, k}^{1}=0$ when $i \neq-1$ in the same way as $Y_{i, k+1}^{1}$. When $i=-1$, we obtain $Y_{-1, k}^{1}=$ $-R_{k-1}$. Similarly, we obtain $Y_{-1, p}^{1}=-R_{p-1}$ when $p=2, \ldots, k-1, Y_{-1, p}^{1}=-R_{p-2}$ when $p=k+2, \ldots, n-1$, and $Y_{i, p}^{1}=0$ otherwise.

Remark 6.8. Eq. (6.23) requires that the $r_{s}$ should be the same for all $s=1, \ldots, n-3$, but the tropicalization of $(6.23)$ is weaker so that the $R_{s}$ can differ.

\section{Duality With BOX-Ball System}

7.1. Basics of the $\mathfrak{s l}_{n}$ box-ball system. The $\mathfrak{s l}_{n}$ box-ball system (BBS) is a cellular automaton, defined as a dynamical system of finitely many balls with $n-1$ 'colors' in an infinite number of boxes arranged along a line. (See [IKT] for a review and a list of 
comprehensive references of the BBS.) The original BBS mentioned in Introduction is the simplest case of $n=2$. In this paper we study the case that $n \geq 3$ and each box can contain one ball at most. By writing 1 for an empty box and $p$ for a box containing a $p$-ball (a ball of color $p$ ) for $p=2, \ldots, n$, we represent a configuration of the system as a (infinite) word on $\{1,2, \ldots, n\}$. The evolution of one time step $t \rightarrow t+1$ is given as follows: Do the following procedure for $p$ from $n$ down to 2 .

(i) Exchange the leftmost $p$ with its nearest 1 to the right.

(ii) Exchange the leftmost $p$ among the rest of the $p$ with its 1 to the right.

(iii) Repeat (ii) until all of the $p$ are moved exactly once.

The resulting word corresponds to the configuration at time $t+1$.

It is known that a soliton of the system is a nonincreasing sequence of $2, \ldots, n$, and that a collection of such sequences gives a multi-soliton solution, in a sense of (i)-(iii) in $\oint 2.4$.

Example 7.1. $n=3$

(i) two solitons:

$$
\begin{aligned}
& t=0: 1133321111321111111111111111 \cdots \\
& t=1: 1111113332113211111111111111 \cdots \\
& t=2: 1111111111332133211111111111 \cdots \\
& t=3: 1111111111111321133321111111 \cdots \\
& t=4: 1111111111111113211113332111 \cdots
\end{aligned}
$$

(ii) three solitons:

$$
\begin{aligned}
t & =0: 113322211113221112111111111111111111111111 \cdots \\
t & =1: 111111133222113221211111111111111111111111 \cdots \\
t & =2: 111111111111332113122222111111111111111111 \cdots \\
t & =3: 111111111111111332311111222221111111111111 \cdots \\
t & =4: 111111111111111111233311111112222211111111 \cdots \\
t & =5: 11111111111111111121133311111111122222111 \cdots
\end{aligned}
$$

The symmetry of the $\mathfrak{s l}_{n}$-BBS is known to be described by the $\hat{\mathfrak{s l}}_{n}$-crystal for the symmetric tensor representation of $U_{q}^{\prime}\left(\hat{\mathfrak{s l}}_{n}\right)$ [FOY, HHIKTT]. Now we present the minimum needed prerequisites concerning the $\hat{\mathfrak{s l}}_{n}$-crystal for the BBS. Recall the $\hat{\mathfrak{s l}}_{n}$-crystal $B_{\ell}$ corresponding to the $\ell$-fold symmetric tensor representation of $U_{q}^{\prime}\left(\hat{\mathfrak{s l}}_{n}\right)$, and given by (3.2) as a set. Let $R_{m \ell}: B_{m} \otimes B_{\ell} \stackrel{\sim}{\rightarrow} B_{\ell} \otimes B_{m}$ be the combinatorial $R$-matrix defined by

$$
R_{m \ell} ; \quad \mathbf{w} \otimes \mathbf{x} \mapsto \mathbf{x}^{\prime} \otimes \mathbf{w}^{\prime},
$$

where

$$
\begin{aligned}
& x_{i}^{\prime}=x_{i}+K_{i+1}-K_{i}, \quad w_{i}^{\prime}=w_{i}+K_{i}-K_{i+1}, \\
& K_{i}:=K_{i}(\mathbf{x}, \mathbf{w})=\min _{j=0,1, \ldots, n-1}\left[\sum_{p=1}^{j} w_{i-p}+\sum_{p=j+2}^{n} x_{i-p}\right] .
\end{aligned}
$$

Here we assume that the subscripts of $w_{i}$ and $x_{i}$ are modulo $n$.

With the notion of a 'carrier' which moves balls, the combinatorial $R$-matrix describes the above time evolution in the following way. The configuration space of the BBS is $B_{1}^{\otimes L}$ for some large number $L$, where $\mathbf{w}=\left(w_{i}\right)_{i=1, \ldots, n} \in B_{1}$ denotes an empty box (resp. a box containing a $p$-ball) when $w_{1}=1$ (resp. $w_{p}=1$ ) and the other $w_{i}$ are zero. A 
carrier of capacity $\ell$ is an element in $B_{\ell}$. Write $\mathbf{w}_{i}^{t} \in B_{1}$ for a state at the $i$-th component of $B_{1}^{\otimes L}$, at time $t$. We assume that the initial carrier $\mathbf{x}_{0}=\left(x_{0, i}\right)_{i=1, \ldots, n} \in B_{\ell}$ has no ball, i.e., $x_{0,1}=\ell$ and the other $x_{0, i}$ are zero, and that $\mathbf{w}_{i}^{t=0}$ for $i \gg 1$ is an empty box. Then the time evolution is given by applying the combinatorial $R$-matrix as

$$
\begin{aligned}
& \mathbf{w}_{L}^{t} \otimes \cdots \otimes \mathbf{w}_{2}^{t} \otimes \mathbf{w}_{1}^{t} \otimes \mathbf{w}_{0}^{t} \otimes \mathbf{x}_{0} \\
& \mapsto \mathbf{w}_{L}^{t} \otimes \cdots \otimes \mathbf{w}_{2}^{t} \otimes \mathbf{w}_{1}^{t} \otimes R_{1 \ell}\left(\mathbf{w}_{0}^{t} \otimes \mathbf{x}_{0}\right)=\mathbf{w}_{L}^{t} \otimes \cdots \otimes \mathbf{w}_{2}^{t} \otimes \mathbf{w}_{1}^{t} \otimes \mathbf{x}_{1}^{t} \otimes \mathbf{w}_{0}^{t+1} \\
& \mapsto \mathbf{w}_{L}^{t} \otimes \cdots \otimes \mathbf{w}_{2}^{t} \otimes R_{1 \ell}\left(\mathbf{w}_{1}^{t} \otimes \mathbf{x}_{1}^{t}\right) \otimes \mathbf{w}_{0}^{t+1}=\mathbf{w}_{L}^{t} \otimes \cdots \otimes \mathbf{w}_{2}^{t} \otimes \mathbf{x}_{2}^{t} \otimes \mathbf{w}_{1}^{t+1} \otimes \mathbf{w}_{0}^{t+1} \\
& \mapsto \quad \cdots \quad=\mathbf{x}_{0} \otimes \mathbf{w}_{L}^{t+1} \otimes \cdots \otimes \mathbf{w}_{2}^{t+1} \otimes \mathbf{w}_{1}^{t+1} \otimes \mathbf{w}_{0}^{t+1}
\end{aligned}
$$

where we denote $R_{1 \ell}\left(\mathbf{w}_{i}^{t} \otimes \mathbf{x}_{i}^{t}\right)=\mathbf{x}_{i+1}^{t} \otimes \mathbf{w}_{i}^{t+1}$ for $i \geq 0$ and set $\mathbf{x}_{0}^{t}=\mathbf{x}_{0}$, at any time $t$. Note that $R_{1 \ell}\left(\mathbf{w} \otimes \mathbf{x}_{0}\right)=\mathbf{x}_{0} \otimes \mathbf{w}$ holds if $\mathbf{w}$ is an empty box, thus we have $\mathbf{x}_{i}^{t}=\mathbf{x}_{0}$ for $i \gg 1$ by the assumption. In the limit $\ell \rightarrow \infty$, the original $\mathfrak{s l}_{n}$-BBS is obtained. We remark that in (7.2), 'right and left' is opposite to that in the original description of the BBS (i)-(iii). We also write the action of the $R$-matrix using a diagram:

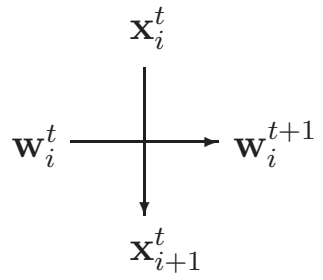

FiguRE 6 . BBS by the combinatorial $R$-matrix

We will use the following lemma later.

Lemma 7.2. Let $\sigma \in S_{n}$ be

$$
\sigma=\left(\begin{array}{ccccc}
1 & 2 & 3 & \cdots & n \\
1 & n & n-1 & \cdots & 2
\end{array}\right)
$$

and let $\rho$ be a map on $\mathbb{Z}^{n} \otimes \mathbb{Z}^{n}$ given by $\left(a_{1}, a_{2}, \ldots, a_{n}\right) \otimes\left(b_{1}, b_{2}, \ldots, b_{n}\right) \mapsto\left(b_{\sigma(1)}, \ldots, b_{\sigma(n)}\right) \otimes$ $\left(a_{\sigma(1)}, \ldots, a_{\sigma(n)}\right)$. Then it holds that $R_{m \ell} \circ \rho=\rho \circ R_{\ell m}$.

We omit the proof, as it is easy. Note that $\rho$ induces a map $B_{m} \otimes B_{\ell} \rightarrow B_{\ell} \otimes B_{m}$ for any $m, \ell$, and that $\rho \circ \rho$ is an identity.

7.2. Observation. Our claim is that the positive soliton solutions of $\Phi(n, k)$ is 'dual' to those of the BBS. Precisely, the dynamics of carriers (resp. states) in $\Phi(n, k)$ for positive solitons coincides with that of states (resp. carriers) of the $\mathfrak{s l}_{n}$-BBS.

Conjecture 7.3. When we have only the positive solitons in $\Phi(n, k)$, the carriers $\mathbf{Z}_{i}^{t}$ take values in a finite set

$$
M:=\left\{\begin{array}{c}
m_{p}:=(\underbrace{1, \ldots, 1}_{p-1}, 0, \underbrace{1, \ldots, 1}_{n-p-1}) ; p=1, \ldots, n-1, \\
m_{n}:=(1,1, \ldots, 1)
\end{array}\right\} \subset\{0,1\}^{n-1} .
$$

Define a map $\beta_{k}: M \rightarrow \tilde{B}_{1}:=\left\{\left(x_{2}, x_{3}, \ldots, x_{n}\right) \in\left(\mathbb{Z}_{\geq 0}\right)^{n-1} ; \sum_{i=2}^{n} x_{i} \leq 1\right\}$ by

$$
m_{p} \mapsto m_{n}-m_{p-1-k}= \begin{cases}(\underbrace{0, \ldots, 0}_{p-2-k}, 1, \underbrace{0, \ldots, 0}_{n-p+k}) & p>k, \\ (\underbrace{0, \ldots, 0}_{n+p-2-k}, 1, \underbrace{0, \ldots, 0}_{-p+k}) & p \leq k,\end{cases}
$$


for $p=1, \ldots, n$ and $k=0,1, \ldots, n-2$, where the subscript $i$ of $m_{i}$ is modulo $n$.

Conjecture 7.4. Assume that the initial configuration $\left(\mathbf{Y}_{i}^{0}\right)_{i}$ of $\Phi(n, k)$ includes only positive solitons. By interchanging space and time coordinates the rules of state and carrier are swapped i.e. $\mathbf{Y}_{i}^{t}$ and $\mathbf{Z}_{i}^{t}$ are respectively regarded as a carrier and a state at time $i$ of space $t$. Then, via the map $\beta_{k}$, the dynamics of the $\mathbf{Z}_{i}^{t}$ is identified with that of the $\mathfrak{s l}_{n}$-BBS where $(0, \ldots, 0) \in \tilde{B}_{1}$ denotes 1 (an empty box) and $(\underbrace{0, \ldots, 0}_{p-2}, 1, \underbrace{0, \ldots, 0}_{n-p}) \in$ $\tilde{B}_{1}$ denotes $p$ (a box containing a $p$-ball) for $p=2, \ldots, n$. If $\left(\mathbf{Y}_{i}^{t}\right)_{i}$ includes a soliton of the minimal form $\left(b_{1}, b_{2}, \ldots, b_{n-1}\right)$ with $b_{k+1}=1$, then the corresponding configuration of BBS includes a soliton as

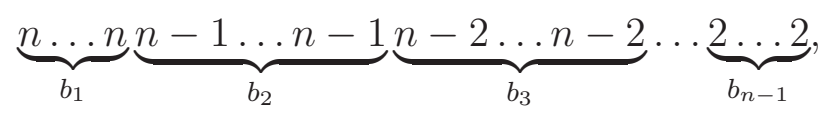

whose velocity is $\sum_{p=1}^{n-1} b_{p}$.

Example 7.5. We show $\left(\mathbf{Z}_{i}^{t}\right)_{i}$ for each $t$, corresponding Example 2.6 and 2.8, Non-initial states are coloured in red. $\Phi(3,1)$ (Example 2.6 (i)):

$$
\begin{aligned}
t & =0:(10)(10)(01)(10)(10)(10)(10)(10)(10)(10)(10)(10) \\
t & =1:(10)(10)(01)(10)(10)(10)(10)(10)(10)(10)(10)(10) \\
t & =2:(10)(10)(01)(10)(10)(10)(10)(10)(10)(10)(10)(10) \\
t & =3:(10)(10)(11)(10)(10)(10)(10)(10)(10)(10)(10)(10) \\
t & =4:(10)(10)(10)(01)(10)(10)(10)(10)(10)(10)(10)(10) \\
t & =5:(10)(10)(10)(01)(10)(10)(10)(10)(10)(10)(10)(10) \\
t & =6:(10)(10)(10)(01)(10)(10)(10)(10)(10)(10)(10)(10) \\
t & =7:(10)(10)(10)(11)(10)(10)(10)(10)(10)(10)(10)(10)
\end{aligned}
$$

$\Phi(4,1)$ (Example 2.6 (ii)):

$$
\begin{aligned}
& t=0:(101)(101)(011)(101)(101)(101)(101)(101)(101)(101) \\
& t=1:(101)(101)(011)(101)(101)(101)(101)(101)(101)(101) \\
& t=2:(101)(101)(011)(101)(101)(101)(101)(101)(101)(101) \\
& t=3:(101)(101)(111)(101)(101)(101)(101)(101)(101)(101) \\
& t=4:(101)(101)(110)(101)(101)(101)(101)(101)(101)(101) \\
& t=5:(101)(101)(110)(101)(101)(101)(101)(101)(101)(101) \\
& t=6:(101)(101)(101)(011)(101)(101)(101)(101)(101)(101) \\
& t=7:(101)(101)(101)(011)(101)(101)(101)(101)(101)(101 \\
& t=8:(101)(101)(101)(011)(101)(101)(101)(101)(101)(101 \\
& t=9:(101)(101)(101)(111)(101)(101)(101)(101)(101)(101) \\
& t=10:(101)(101)(101)(110)(101)(101)(101)(101)(101)(101)
\end{aligned}
$$


$\Phi(3,1)$ (Example 2.8 (i)):

$$
\begin{aligned}
t & =0:(10)(10)(01)(10)(01)(10)(10)(10)(10)(10)(10)(10) \\
t & =1:(10)(10)(11)(10)(01)(10)(10)(10)(10)(10)(10)(10) \\
t & =2:(10)(10)(10)(01)(11)(10)(10)(10)(10)(10)(10)(10) \\
t & =3:(10)(10)(10)(11)(10)(01)(10)(10)(10)(10)(10)(10) \\
t & =4:(10)(10)(10)(10)(01)(11)(10)(10)(10)(10)(10)(10) \\
t & =5:(10)(10)(10)(10)(01)(10)(01)(10)(10)(10)(10)(10) \\
t & =6:(10)(10)(10)(10)(11)(10)(11)(10)(10)(10)(10)(10) \\
t & =7:(10)(10)(10)(10)(10)(01)(10)(01)(10)(10)(10)(10) \\
t & =8:(10)(10)(10)(10)(10)(01)(10)(11)(10)(10)(10)(10) \\
t & =9:(10)(10)(10)(10)(10)(01)(10)(10)(01)(10)(10)(10) \\
t & =10:(10)(10)(10)(10)(10)(11)(10)(10)(11)(10)(10)(10)
\end{aligned}
$$

The last case is dual with Example 7.1 (i).

Remark 7.6. The positive solitons for $\Phi(n, k)$ do not correspond to all $\mathfrak{s l}_{n}$-BBS solitons; a BBS soliton related to a positive soliton of $\Phi(n, k)$ should include at least one $p$-ball for $p \in\{2, \ldots, n\} \backslash\{n-k\}$, and exactly one $(n-k)$-ball.

7.3. Strategy to prove Conjectures $\mathbf{7 . 3}$ and $\mathbf{7 . 4}$. Let $\tilde{B}_{\ell}$ be a set as

$$
\tilde{B}_{\ell}=\left\{\mathbf{x}=\left(y_{1}, y_{2}, \ldots, y_{n-1}\right) \in\left(\mathbb{Z}_{\geq 0}\right)^{n-1} ; \sum_{i=1}^{n} y_{i} \leq \ell\right\} .
$$

We have a natural isomorphism $\gamma_{\ell}: B_{\ell} \rightarrow \tilde{B}_{\ell}$ given by $\left(x_{1}, x_{2}, \ldots, x_{n}\right) \mapsto\left(x_{2}, x_{3}, \ldots, x_{n}\right)$, where the inverse map $\gamma_{\ell}^{-1}$ is given by $\left(y_{1}, y_{2}, \ldots, y_{n-1}\right) \mapsto\left(\ell-\sum_{i=1}^{n-1} y_{i}, y_{1}, \ldots, y_{n-1}\right)$. Define $\tilde{R}_{m \ell}$ as

$$
\tilde{R}_{m \ell}:=\left(\gamma_{\ell} \otimes \gamma_{m}\right) \circ R_{m \ell} \circ\left(\gamma_{m}^{-1} \otimes \gamma_{\ell}^{-1}\right): \tilde{B}_{m} \otimes \tilde{B}_{\ell} \stackrel{\sim}{\rightarrow} \tilde{B}_{\ell} \otimes \tilde{B}_{m} .
$$

Recall the maps $\iota$ on $\mathbb{R}^{n-1}$ and $\tilde{\rho}$ on $\left(\mathbb{R}^{n-1}\right)^{2}$ defined in Section 3 . We use the same notations $\iota$ and $\tilde{\rho}$ for their restrictions on $\mathbb{Z}$. We identify $\left(\mathbb{Z}^{n-1}\right)^{2}$ with $\mathbb{Z}^{n-1} \otimes \mathbb{Z}^{n-1}$, following the expression of the combinatorial $R$-matrix.

Lemma 7.7. The followings hold:

(i) $\left(\gamma_{\ell} \otimes \gamma_{m}\right) \circ \rho \circ\left(\gamma_{m}^{-1} \otimes \gamma_{\ell}^{-1}\right)=\tilde{\rho}$.

(ii) $\tilde{\rho} \circ \tilde{R}_{m \ell} \circ \tilde{\rho}=\tilde{R}_{\ell m}$ on $\tilde{B}_{\ell} \otimes \tilde{B}_{m}$.

(iii) $\tilde{\rho} \circ\left(\beta_{k} \otimes \iota\right)=\left(\iota \otimes \beta_{n-2-k}\right) \circ \tilde{\rho}$ on $M \otimes \tilde{B}_{m}$, for $k=0,1, \ldots, n-2-k$.

Proof. (i) is easy. (ii) follows from (i) and Lemma 7.2. We check (iii). For $m_{p} \otimes \mathbf{w} \in$ $M \otimes \tilde{B}_{m}$, we have

$$
\begin{aligned}
& \left(\iota \otimes \beta_{n-2-k}\right) \circ \tilde{\rho}\left(m_{p} \otimes \mathbf{w}\right)=\left(\iota \otimes \beta_{n-2-k}\right)\left(\iota(\mathbf{w}) \otimes m_{n-p}\right)=\mathbf{w} \otimes\left(m_{n}-m_{k+1-p}\right), \\
& \tilde{\rho} \circ\left(\beta_{k} \otimes \iota\right)\left(m_{p} \otimes \mathbf{w}\right)=\tilde{\rho}\left(\left(m_{n}-m_{p-1-k}\right) \otimes \iota(\mathbf{w})\right)=\mathbf{w} \otimes\left(m_{n}-m_{-p+1+k}\right),
\end{aligned}
$$

for $p=1, \ldots, n$ and $k=0,1, \ldots, n-2-k$.

As with $\phi(n, k)$ in Section 3, we use the same notation $\Phi(n, k)$ to denote the map on $\mathbb{Z}^{n-1} \otimes \mathbb{Z}^{n-1}$ which is the building block of the dynamics $\Phi(n, k)$. We write $\Phi(n, k)$ : $\mathbf{Z}_{i}^{t} \otimes \mathbf{Y}_{i}^{t} \mapsto \mathbf{Y}_{i}^{t+1} \otimes \mathbf{Z}_{i+1}^{t}$, with a diagram, 


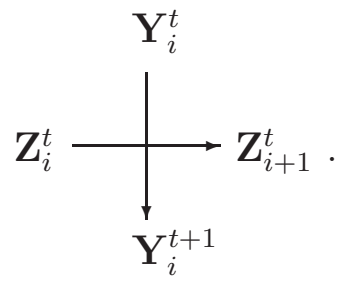

Proposition 7.8. If it holds that

$$
\tilde{R}_{1 \infty} \circ\left(\beta_{k} \otimes \iota\right)\left(m_{p} \otimes \mathbf{w}\right)=\left(\iota \otimes \beta_{k}\right) \circ \Phi(n, k)\left(m_{p} \otimes \mathbf{w}\right)
$$

for some $k \in\{0,1, \ldots, n-2\}$ and some $m_{p} \otimes \mathbf{w} \in M \otimes \tilde{B}_{\infty}$, then

$$
\tilde{R}_{1 \infty} \circ\left(\beta_{n-2-k} \otimes \iota\right)\left(m_{n-p^{\prime}} \otimes \iota\left(\mathbf{w}^{\prime}\right)\right)=\left(\iota \otimes \beta_{n-2-k}\right) \circ \Phi(n, n-2-k)\left(m_{n-p^{\prime}} \otimes \iota\left(\mathbf{w}^{\prime}\right)\right),
$$

where $\left(\mathbf{w}^{\prime} \otimes m_{p}^{\prime}\right):=\Phi(n, k)\left(m_{p} \otimes \mathbf{w}\right)$.

Proof. Eq. (7.3) can be rewritten as

$$
\tilde{R}_{1 \infty} \circ\left(\beta_{k} \otimes \iota\right) \Phi(n, k)^{-1}\left(\mathbf{w}^{\prime} \otimes m_{p}^{\prime}\right)=\left(\iota \otimes \beta_{k}\right)\left(\mathbf{w}^{\prime} \otimes m_{p}^{\prime}\right) .
$$

By tropicalizing (3.3), it holds that

$$
\Phi(n, n-2-k)=\tilde{\rho} \circ \Phi^{-1}(n, k) \circ \tilde{\rho}
$$

on $\mathbb{Z}^{n-1} \otimes \mathbb{Z}^{n-1}$. By using this and Lemma 7.7, the 1.h.s. of the first equation becomes

$$
\begin{aligned}
& \tilde{\rho} \circ \tilde{R}_{\infty 1} \circ \tilde{\rho} \circ\left(\beta_{k} \otimes \iota\right) \circ \tilde{\rho} \circ \Phi(n, n-2-k) \circ \tilde{\rho}\left(\mathbf{w}^{\prime} \otimes m_{p}^{\prime}\right) \\
& \quad=\tilde{\rho} \circ \tilde{R}_{\infty 1} \circ\left(\iota \otimes \beta_{n-2-k}\right) \Phi(n, n-2-k)\left(m_{n-p^{\prime}} \otimes \iota\left(\mathbf{w}^{\prime}\right)\right) .
\end{aligned}
$$

On the other hand, the r.h.s. becomes $\tilde{\rho} \circ\left(\beta_{n-2-k} \otimes \iota\right)\left(m_{n-p^{\prime}} \otimes \iota\left(\mathbf{w}^{\prime}\right)\right)$. Thus the claim follows.

To prove Conjectures 7.3 and 7.4 we have to check that all configurations which appear in propagating positive solitons have the form $m_{p} \otimes \mathbf{w} \mapsto \mathbf{w}^{\prime} \otimes m_{p^{\prime}}$ and satisfy (7.3). Proposition 7.8 means that the claims in the conjectures for $\Phi(n, n-2-k)$ follow from those for $\Phi(n, k)$. In the next two subsections, we prove these conjectures in the cases of $n=3$ and 4 .

7.4. Proof for $\Phi(3, k)$. We prove the case of $\Phi(3,1)$, from which the case of $\Phi(3,0)$ follows due to Proposition 7.8 .

We say a finite sequence of states is stable when the carrier returns to its initial state after passing through the sequence. For example, in the case of $\Phi(3,1)$ the vacuum state $(0,0)$ is stable, and a sequence $(3,1)(2,0)$ is stable but $(3.1)(2,1)$ is not, as shown by diagrams:

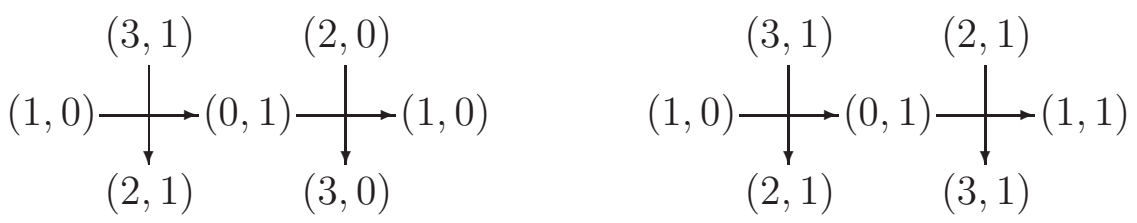

Lemma 7.9. The following sequences of states are stable:

(a) $(i, 1)(0,0) ; i>0$,

(b) $(i, 1)(j, 0) ; i, j>0$, 
(c) $(0,1)(i, 0) ; i>0$,

(d) $(i, 1)\left(j^{\prime}, 1\right)(k, 0) ; i, k>0, j^{\prime} \geq 0$,

Assume that the initial configuration of $\left(\mathbf{Y}_{i}^{t=0}\right)_{i}$ for $\Phi(3,1)$ consists of the vacuum state $(0,0)$ and a finite number of the above sequences. Then the configuration for $t=1$ again consist of the vacuum state and these sequences.

Proof. From (2.11) we see that all sequences (a)-(d) are stable by the diagrams in Figure 7. Note that the vacuum state $(0,0)$ is also stable:

$$
(1,0) \otimes(0,0) \mapsto(0,0) \otimes(1,0) .
$$

When the configuration at $t=0$ is given by a composition of these stable sequences, the configuration at $t=1$ is obtained by simply combining the diagrams in Figure 7 , due to the stability. It turns out that (a) and (b) change to the form of (b) or (c), (c) changes to the form of $(0,0)(a)^{*}$, and $(d)$ changes to the form of $(b)(a)^{*}$ or $(c)(a)^{*}$. Here we define $(\mathrm{a})^{*}:=(i, 1)$ for $i>0$. Thus, at $t=1$ the sequence immediately to the right of $(\mathrm{a})^{*}$ is always $(0,0),(\mathrm{b})$ or $(\mathrm{c})$, but not $(\mathrm{a})$. If it is $(0,0)$, we obtain the form of (a). If it is (b) or (c), we obtain the form of (d). Since the number of (a) $-(d)$ at $t=0$ is finite, all (a)* which appear at $t=1$ turn out to be a part of a new (a) or (d). Then the claim follows.

(a)<smiles>C1=[GeH][AsH]C1</smiles>

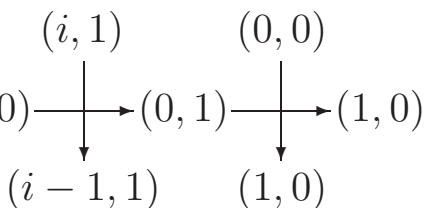

(b)

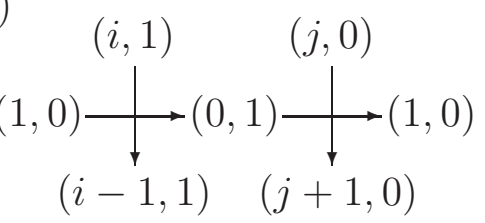

(c)

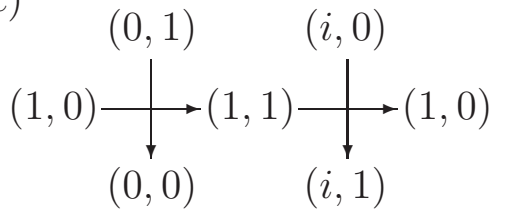

(d)

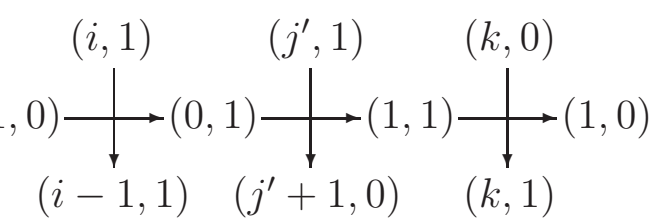

Figure 7 . The stable sequences for $\Phi(3,1)\left(i, j, k>0, j^{\prime} \geq 0\right)$.

The following is easily seen from Figure 8 ,

Lemma 7.10. A soliton corresponds to a sequence of the form (a)-(c). The soliton at its minimal length has the form $(a)$, i.e. it equals $(k, 1)$ for some $k>0$, The velocity of the soliton is $1 /(k+1)$.

Proof of Conjecture 7.3 for $\Phi(3,1)$. Assume that we start with an initial configuration including $N>1$ sequences of forms (a)-(c), which is an $N$-soliton state. Then a faster soliton catches up with a slower one, and overtakes it after some scattering states (d). Finally, the $N$ solitons line up in a way that slower ones are left and faster ones are right. Thus, from Figure [7, the possible configurations which appear in propagating positive solitons are as Figure 9, where we have only $(1,0),(1,1)$ and $(0,1)$ for the carriers.

Proof of Conjecture 7.4 for $\Phi(3,1)$. In Figure 8 , we see that during rightward propagation of a soliton $(k, 1)$, a sequence of carriers $\left(\mathbf{Z}_{1}^{t}\right)_{t=0,1, \ldots, k}=(\underbrace{(0,1), \ldots,(0,1)}_{k},(1,1))$ 


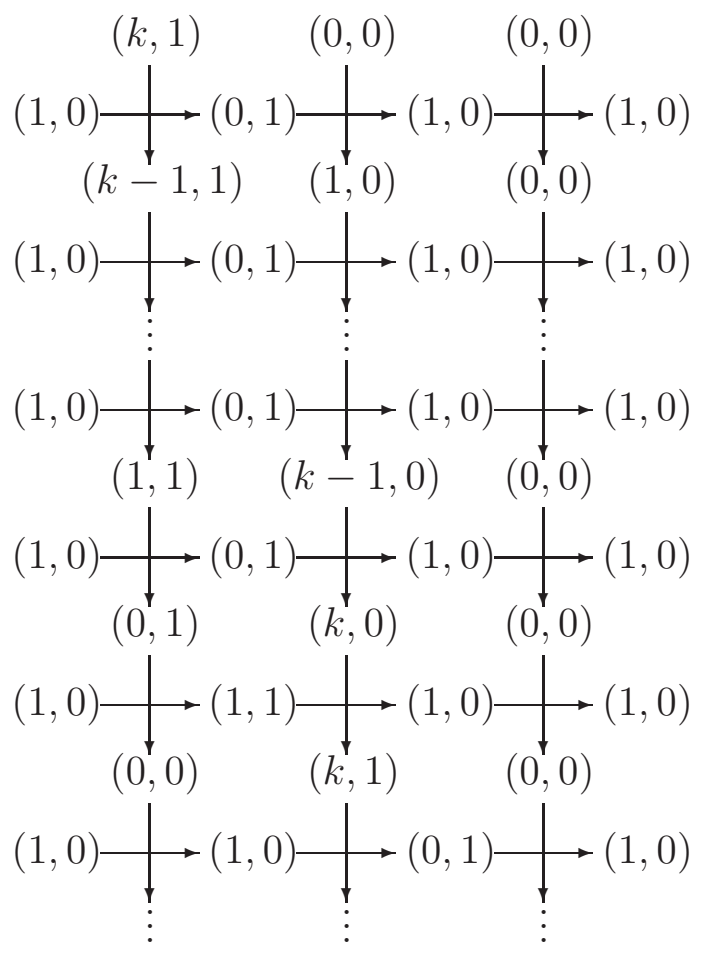

Figure 8. Propagation of a soliton.

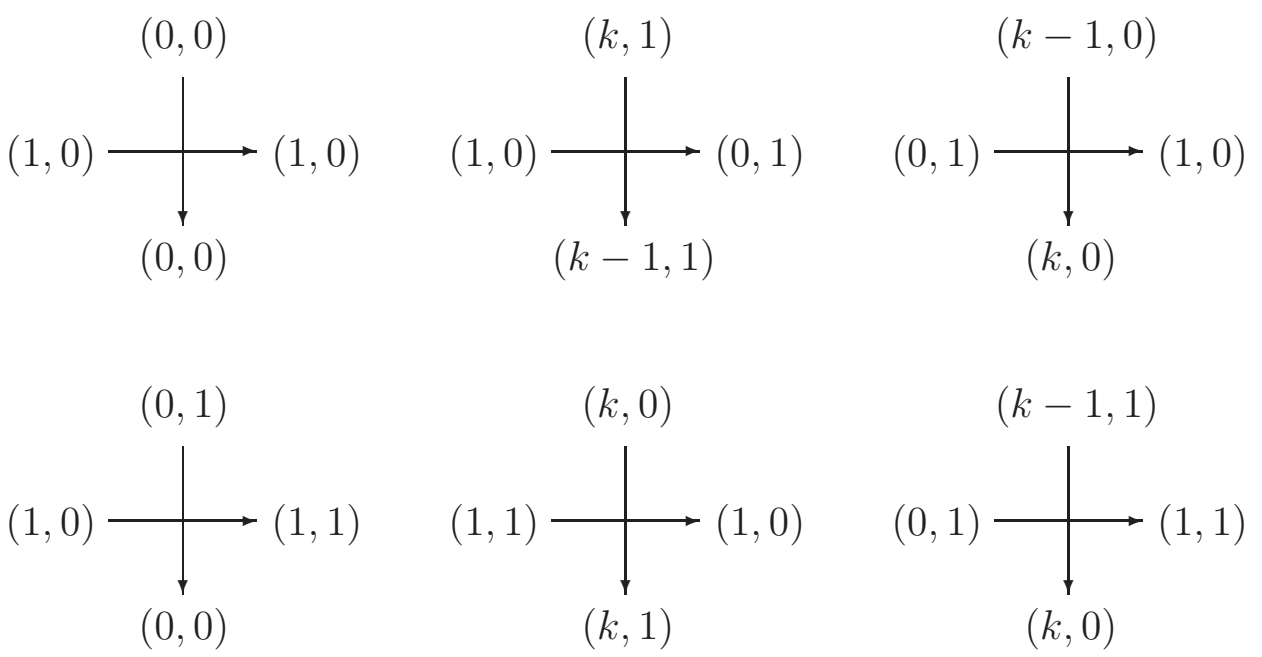

Figure 9. Possible configurations to propagate solitons in $\Phi(3,1)\left(k \in \mathbb{Z}_{\geq 1}\right)$.

propagates downward with velocity $k+1$. By the map $\beta_{1}$, this sequence is transformed into $(\underbrace{(0,1), \ldots,(0,1)}_{k},(1,0))$ which corresponds to a soliton $\underbrace{33 \cdots 3}_{k} 2$ of the $\mathfrak{s l}_{3}$-BBS.

The configurations in Figure 9 are the local diagrams appearing in Figure 7, which are nothing but those that appear in soliton propagations. We transform them into the diagrams in Figure 10, by acting by $\beta_{1}$ on carriers and $\iota$ on states. Using (7.1), one sees that (7.3) is fulfilled by $m_{p} \otimes \mathbf{w} \in M \otimes \tilde{B}_{\infty}$ appearing in Figure 9. It turns out that all configurations in Figure 10 are what appear when $R_{1 \infty}$ propagates solitons of the form $\underbrace{33 \cdots 3}_{k>0} 2$, due to the following facts: in the states $\left(x_{2}, x_{3}\right)$ on the vertical 
edges in Figure 10, $x_{2}$ takes only 0 or 1 which means that each soliton includes at most one 2-ball. There is neither configuration $\tilde{R}_{1 \infty}((0,0) \otimes(0,1))=(0,0) \otimes(0,1)$ nor $\tilde{R}_{1 \infty}((1,0) \otimes(0,0))=(1,0) \otimes(0,0)$. This means that there is neither a soliton containing only 3 -balls, nor a soliton containing only 2 -balls.

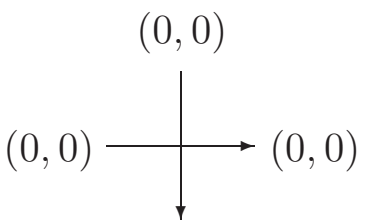

$(0,0)$

$(0,0)$<smiles>CC(C)(C)[O-]</smiles>

$(1,0)$<smiles>CC(C)(C)[O-]</smiles>

$(1, k-1)$

$(0, k)$<smiles>CC(C)(C)[O-]</smiles>

$(1, k)$

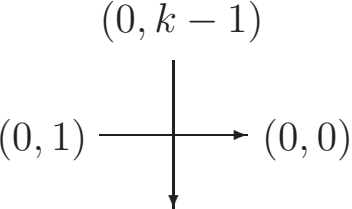

$(0, k)$

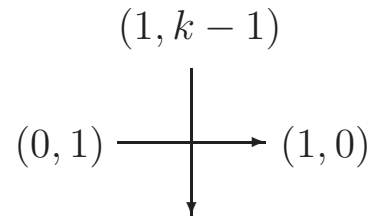

$(0, k)$

Figure 10. The $\mathfrak{s l}_{3}$-BBS configurations from $\Phi(3,1)\left(k \in \mathbb{Z}_{\geq 1}\right)$.

7.5. Proof for $\Phi(4, k)$. First we consider $\Phi(4,2)$. The map $\phi(4,2)$ is given by

$$
\begin{aligned}
\left(z_{i, 1}^{t}, z_{i, 2}^{t}, z_{i, 3}^{t}\right) \otimes\left(y_{i, 1}^{t}, y_{i, 2}^{t}, y_{i, 3}^{t}\right) & \\
& \mapsto\left(y_{i, 1}^{t+1}, y_{i, 2}^{t+1}, y_{i, 3}^{t+1}\right) \otimes\left(z_{i+1,1}^{t}, z_{i+1,2}^{t}, z_{i+1,3}^{t}\right) \\
& =\left(\frac{z_{i, 3}^{t} y_{i, 1}^{t}}{z_{i, 2}^{t}+y_{i, 1}^{t}}, \frac{\left(z_{i, 2}^{t}+y_{i, 1}^{t}\right) y_{i, 2}^{t}}{z_{i, 1}^{t}+y_{i, 2}^{t}}, z_{i, 1}^{t}+y_{i, 2}^{t}\right) \otimes\left(\frac{z_{i, 1}^{t}\left(z_{i, 2}^{t}+y_{i, 1}^{t}\right)}{z_{i, 1}^{t}+y_{i, 2}^{t}}, \frac{z_{i, 2}^{t} z_{i, 3}^{t}}{z_{i, 2}^{t}+y_{i, 1}^{t}}, y_{i, 3}^{t}\right) .
\end{aligned}
$$

Lemma 7.11. The following sequences of states are stable:

(a1) $(i, j, 1)(0,0,0) ; i, j>0$,

(a2) $\left(i_{1}, j, 1\right)\left(i_{2}, 0,0\right) ; i_{1}, i_{2}, j>0$,

(b1) $(0, j, 1)(i, 0,0) ; i, j>0$,

(b2) $(0, j, 1)\left(i, j^{\prime}, 0\right) ; i, j_{1}, j_{2}>0$,

(c) $(0,0,1)(i, j, 0) ; i, j>0$,

(d) $\left(0, j_{1}, 1\right)\left(i_{1}, j^{\prime}, 1\right)\left(i_{2}, j_{2}, 0\right) ; i_{1}, i_{2}, j_{1}, j_{2}>0, j^{\prime} \geq 0$,

(e1) $\left(i_{1}, j_{1}, 1\right)\left(i^{\prime}, j_{2}, 1\right)\left(i_{2}, j^{\prime}, 0\right) ; i_{1}, i_{2}, j_{1}, j_{2}>0, i^{\prime}, j^{\prime} \geq 0$

(e2) $\left(i_{1}, j_{1}, 1\right)\left(i^{\prime}, 0,1\right)\left(i_{2}, j_{2}, 0\right) ; i_{1}, i_{2}, j_{1}, j_{2}>0, i^{\prime} \geq 0$

(f) $\left(i_{1}, j_{1}, 1\right)\left(i^{\prime}, j_{2}, 1\right)\left(i_{2}, j^{\prime}, 1\right)\left(i_{3}, j_{3}, 0\right) ; i_{1}, i_{2}, i_{3}, j_{1}, j_{2}, j_{3}>0, i^{\prime}, j^{\prime} \geq 0$.

Assume that the initial configuration of $\left(\mathbf{Y}_{i}^{t=0}\right)_{i}$ for $\Phi(4,2)$ consists of the vacuum state $(0,0,0)$ and a finite number of these sequences of states. Then the configuration for $t=1$ again consist of the vacuum state and these sequences.

Proof. It is shown in the same way as in the case of $\Phi(3,1)$ : due to the map $\Phi(4,2)$ given by the tropicalization of (7.6), we obtain diagrams in Figure 11, and see that all sequences (a1)-(f) and the vacuum state $(0,0,0)$ are stable. Then one sees that (a1) changes to (a2), (a2) changes to (a2) or (b1), (b1) changes to (b2) or (c), (c) changes to 
$(0,0,0)(\mathrm{a})^{*},(\mathrm{~d})$ changes to $(\mathrm{b} 2)(\mathrm{a})^{*}$ or $(\mathrm{c})(\mathrm{a})^{*},(\mathrm{e} 1)$ changes to $(\mathrm{e} 1)$ or $(\mathrm{d})$ or $(\mathrm{e} 2),(\mathrm{e} 2)$ changes to $(\mathrm{a} 2)(\mathrm{a})^{*}$, and $(\mathrm{f})$ changes to $(\mathrm{e} 1)(\mathrm{a})^{*}$ or $(\mathrm{d})(\mathrm{a})^{*}$. Moreover, $(\mathrm{a})^{*}(0,0,0)$ has a form of $(\mathrm{a} 1)$, and $(\mathrm{a})^{*}(\mathrm{x})$ has a form of $(\mathrm{e} 1)$ or $(\mathrm{e} 2)$ or $(\mathrm{f})$ where $(\mathrm{x})$ is one of $(\mathrm{a} 2)-(\mathrm{e} 2)$. Then the claim follows.

(a1)

$(1,1,0)$

$$
(i, j, 1) \quad(0,0,0)
$$

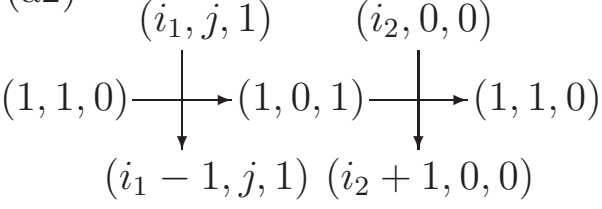

(b1)

$$
(0, j, 1) \quad(i, 0,0)
$$

$(1,1,0)$

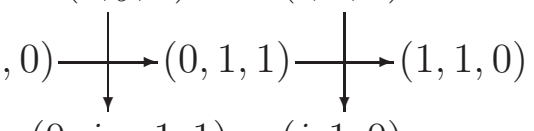

$$
(0, j-1,1) \quad(i, 1,0)
$$

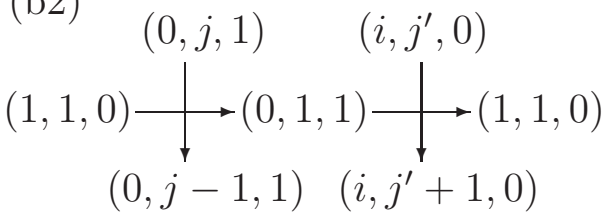

(c)

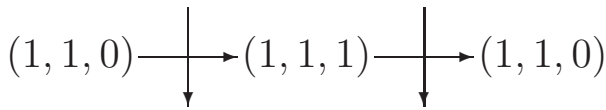

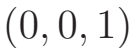<smiles>[Mg][Mg]</smiles>

(d)

$$
(0,0,0) \quad(i, j, 1)
$$

$$
\left(0, j_{1}, 1\right) \quad\left(i_{1}, j^{\prime}, 1\right) \quad\left(i_{2}, j_{2}, 0\right)
$$

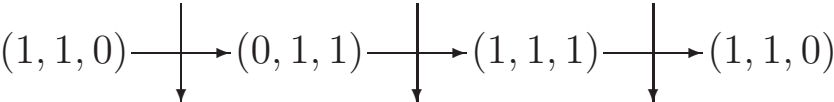

$\left(0, j_{1}-1,1\right) \quad\left(i_{1}, j+1,0\right) \quad\left(i_{2}, j_{2}, 1\right)$

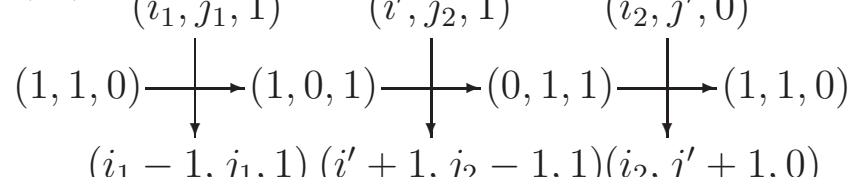

$(\mathrm{e} 2)$

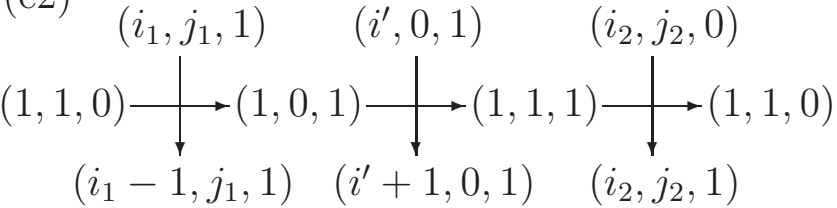

(f)

$$
\left(i_{1}, j_{1}, 1\right) \quad\left(i^{\prime}, j_{2}, 1\right) \quad\left(i_{2}, j^{\prime}, 1\right) \quad\left(i_{3}, j_{3}, 0\right)
$$

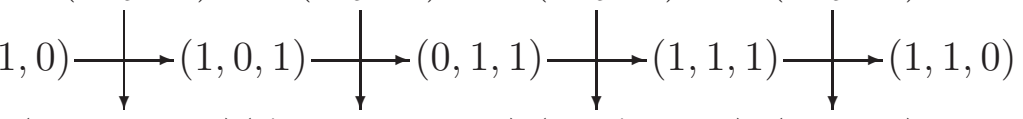

$\left(i_{1}-1, j_{1}, 1\right)\left(i^{\prime}+1, j_{2}-1,1\right)\left(i_{2}, j^{\prime}+1,0\right)\left(i_{3}, j_{3}, 1\right)$

Figure 11. Carrier stable sequences for $\Phi(4,2)\left(i, j, i_{*}, j_{*}>0, i^{\prime}, j^{\prime} \geq 0\right)$.

Proof of Conjecture 7.3 and 7.4 for $\Phi(4,2)$. We give an outline of the proof. It is easy to show that one-soliton propagation is given by combining diagrams (a1)-(c) in Figure 11, thus a soliton of the minimal form $(i, j, 1)$ has velocity $1 /(i+j+1)$. Further, from Lemma 7.11 it follows that the propagation of any multi-soliton state is described by diagrams (a1)-(f) and

$$
(1,1,0) \otimes(0,0,0) \mapsto(0,0,0) \otimes(1,1,0),
$$

so Conjecture 7.3 follows. The diagrams in Figure 11 consist of 'local' diagrams as in Figure 12 and (7.7), and they are shown to satisfy (7.3). Then we see that in the corresponding $\mathfrak{s l}_{4}$-BBS only solitons of forms as $\underbrace{4 \cdots 4}_{i} \underbrace{3 \cdots 3}_{j} 2$ for some $i, j>0$ appear, which proves Conjecture 7.4 .

Conjectures 7.3 and 7.4 for $\Phi(4,0)$ follows from the above result on $\Phi(4,2)$ and Proposition [7.8, where the corresponding $\mathfrak{s l}_{4}$-BBS only includes solitons of forms as $4 \underbrace{3 \cdots 3}_{i} \underbrace{2 \cdots 2}_{j}$ for some $i, j>0$. 


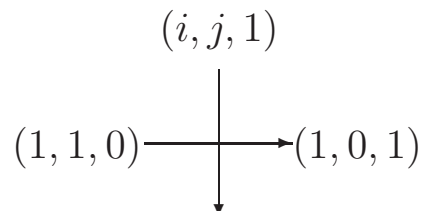

$(i-1, j-1,1)$

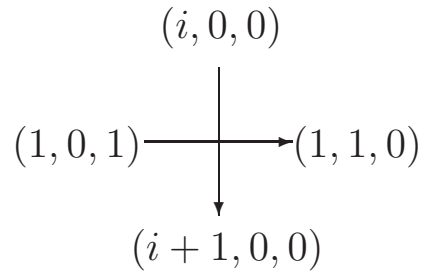

$$
(i, j-1,0)
$$

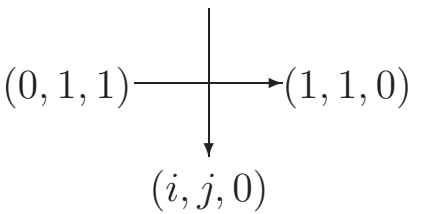

$(0, j, 1)$<smiles>CCCC(C)(C)CC</smiles>

$(0, j-1,1)$

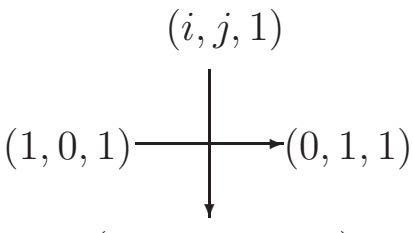

$(i+1, j-1,1)$

$(i, j-1,1)$

$(0,1,1)$

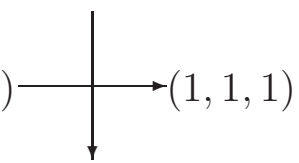

$(i, j, 1)$
$(0,0,1)$

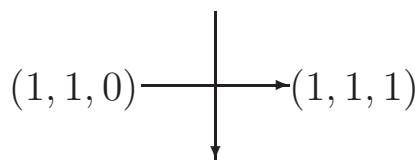

$(0,0,0)$

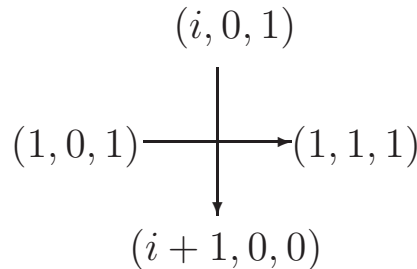

$(i, j, 0)$

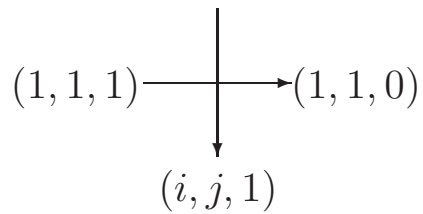

Figure 12. Possible configurations to propagate solitons for $\Phi(4,2)$ $\left(i, j \in \mathbb{Z}_{\geq 1}\right)$.

Next we consider $\Phi(4,1)$.

Lemma 7.12. The following sequences of states are stable:

(a1) $(i, 1, j)(0,0,0) ; i, j>0$

(a2) $\left(i_{1}, 1, j\right)\left(i_{2}, 0,0\right) ; i_{1}, i_{2}, j>0$

(b) $(0,1, j)(i, 0,0) ; i, j>0$,

(c1) $(0,0, j)(i, 1,0) ; i, j>0$,

(c2) $\left(0,0, j_{1}\right)\left(i, 1, j_{2}\right) ; i, j_{1}, j_{2}>0$,

(d) $\left(0,1, j_{1}\right)\left(i_{1}, 0, j_{2}\right)\left(i_{2}, 1, j^{\prime}\right) ; i_{1}, i_{2}, j_{1}, j_{2}>0, j^{\prime} \geq 0$,

(e1) $\left(i_{1}, 1, j_{1}\right)\left(i^{\prime}, 1, j_{2}\right)\left(i_{2}, 0,0\right) ; i_{1}, i_{2}, j_{1}, j_{2}>0, i^{\prime} \geq 0$

(e2) $\left(i_{1}, 1, j_{1}\right)\left(i^{\prime}, 0, j_{2}\right)\left(i_{2}, 1, j^{\prime}\right) ; i_{1}, i_{2}, j_{1}, j_{2}>0, i^{\prime}, j^{\prime} \geq 0$,

(f) $\left(i_{1}, 1, j_{1}\right)\left(i^{\prime}, 1, j_{2}\right)\left(i_{2}, 0, j_{3}\right)\left(i_{3}, 1, j^{\prime}\right) ; i_{1}, i_{2}, i_{3}, j_{1}, j_{2}, j_{3}>0, i^{\prime}, j^{\prime} \geq 0$.

Assume that the initial configuration of $\left(\mathbf{Y}_{i}^{t=0}\right)_{i}$ for $\Phi(4,1)$ consists of the vacuum state $(0,0,0)$ and a finite number of these sequences. Then the configuration for $t=1$ again consist of the vacuum state and these sequences.

Using this lemma, one can show that Conjectures 7.3 and 7.4 holds for $\Phi(4,1)$, in the same manner as the case of $\Phi(4,2)$. The corresponding $\mathfrak{s l}_{4}$-BBS only includes solitons of forms as $\underbrace{4 \cdots 4}_{i} 3 \underbrace{2 \cdots 2}_{j}$ for some $i, j>0$. 
8. Numerical PHENOMEnA: NEGATIVE SOlitons, RElAXATION SOlitons, AND PULSARS

Besides positive solitons, we numerically observe negative solitons, relaxation solitons and pulsars for $\Phi(n, k)$ on $\mathbb{Z}$ with the commuting pair given by (2.12). It might be an interesting future problem to study these phenomena.

8.1. Negative solitons. For the definition of a negative soliton, see 92.4 . We observe that a state $(\underbrace{-p, \ldots,-p}_{n-1})$ for $p \in \mathbb{Z}_{>0}$ is a negative soliton for $\Phi(n, k)$, whose velocity is $1 /(n-1)$ independent of $p$. The difference among the negative solitons appears in scattering with positive solitons. The following examples show that in scatterings of positive and negative solitons the phase shift of the positive soliton depends on $p$.

Example 8.1. Scatterings of positive and negative solitons in the case of $\Phi(3,1)$. We write $\bar{k}$ for $-k$, for $k \in \mathbb{Z}_{>0}$.

(i) $(\overline{1}, \overline{1}) \times(3,1) \mapsto(3,1) \times(\overline{1}, \overline{1})$ :

$$
\begin{aligned}
t & =0:(00)(\overline{1} \overline{1})(00)(31)(00)(00)(00)(00)(00)(00)(00)(00) \\
t & =1:(00)(0 \overline{1})(\overline{1} 0)(21)(10)(00)(00)(00)(00)(00)(00)(00) \\
t & =2:(00)(00)(\overline{1} \overline{1})(11)(20)(00)(00)(00)(00)(00)(00)(00) \\
t & =3:(00)(00)(0 \overline{1})(\overline{1} 1)(30)(00)(00)(00)(00)(00)(00)(00) \\
t & =4:(00)(00)(00)(\overline{1} \overline{1})(31)(00)(00)(00)(00)(00)(00)(00) \\
t & =5:(00)(00)(00)(0 \overline{1})(02)(2 \overline{1})(00)(00)(00)(00)(00)(00) \\
t & =6:(00)(00)(00)(00)(\overline{1} 0)(40)(\overline{1} 0)(00)(00)(00)(00)(00) \\
t & =7:(00)(00)(00)(00)(0 \overline{1})(22)(0 \overline{1})(00)(00)(00)(00)(00) \\
t & =8:(00)(00)(00)(00)(00)(01)(3 \overline{1})(\overline{1} 0)(00)(00)(00)(00) \\
t & =9:(00)(00)(00)(00)(00)(00)(31)(\overline{1} \overline{1})(00)(00)(00)(00) \\
t & =10:(00)(00)(00)(00)(00)(00)(21)(1 \overline{1})(\overline{1} 0)(00)(00)(00) \\
t & =11:(00)(00)(00)(00)(00)(00)(11)(20)(\overline{1} \overline{1})(00)(00)(00) \\
t & =12:(00)(00)(00)(00)(00)(00)(01)(30)(0 \overline{1})(\overline{1} 0)(00)(00) \\
t & =13:(00)(00)(00)(00)(00)(00)(00)(31)(00)(\overline{1} \overline{1})(00)(00)
\end{aligned}
$$


(ii) $(\overline{2}, \overline{2}) \times(3,1) \mapsto(3,1) \times(\overline{2}, \overline{2})$ :

$$
\begin{aligned}
t & =0:(00)(\overline{2} \overline{2})(00)(31)(00)(00)(00)(00)(00)(00)(00)(00) \\
t & =1:(00)(0 \overline{2})(\overline{2} 0)(21)(10)(00)(00)(00)(00)(00)(00)(00) \\
t & =2:(00)(00)(\overline{2} \overline{2})(11)(20)(00)(00)(00)(00)(00)(00)(00) \\
t & =3:(00)(00)(0 \overline{2})(\overline{2} 1)(30)(00)(00)(00)(00)(00)(00)(00) \\
t & =4:(00)(00)(00)(\overline{2} \overline{2})(31)(00)(00)(00)(00)(00)(00)(00) \\
t & =5:(00)(00)(00)(0 \overline{2})(\overline{2} 3)(3 \overline{2})(00)(00)(00)(00)(00)(00) \\
t & =6:(00)(00)(00)(00)(\overline{2} \overline{2})(51)(\overline{2} 0)(00)(00)(00)(00)(00) \\
t & =7:(00)(00)(00)(00)(0 \overline{2})(03)(1 \overline{2})(00)(00)(00)(00)(00) \\
t & =8:(00)(00)(00)(00)(00)(\overline{2} 0)(5 \overline{1})(\overline{2} 0)(00)(00)(00)(00) \\
t & =9:(00)(00)(00)(00)(00)(0 \overline{2})(23)(\overline{1} \overline{2})(00)(00)(00)(00) \\
t & =10:(00)(00)(00)(00)(00)(00)(\overline{1} 1)(4 \overline{2})(\overline{2} 0)(00)(00)(00) \\
t & =11:(00)(00)(00)(00)(00)(00)(0 \overline{1})(32)(\overline{2} \overline{2})(00)(00)(00) \\
t & =12:(00)(00)(00)(00)(00)(00)(00)(11)(2 \overline{2})(\overline{2} 0)(00)(00) \\
t & =13:(00)(00)(00)(00)(00)(00)(00)(01)(30)(\overline{2} \overline{2})(00)(00) \\
t & =14:(00)(00)(00)(00)(00)(00)(00)(00)(31)(0 \overline{2})(\overline{2} 0)(00) \\
t & =15:(00)(00)(00)(00)(00)(00)(00)(00)(21)(10)(\overline{2} \overline{2})(00)
\end{aligned}
$$

Remark 8.2. Negative solitons for the $\mathfrak{s l}_{2}$-BBS were found by Hirota $\mathrm{H} 2$, and studied in [KMT, WNSRG and others. In [KMT], it is clarified that the states with negative solitons are transformed into the $\mathfrak{s l}_{2}$-BBS with greater box capacity. It is not clear for now if some similar mechanism works in the general $\mathfrak{s l}_{n}$-BBS or in $\Phi(n, k)$.

8.2. Relaxation solitons and pulsars. Besides solitons, we introduce two phenomena, relaxation solitons and pulsars, which may satisfy the condition (i) for solitons presented in 92.4 , but not (ii).

We define a relaxation soliton as a finite sequence of non-vacuum states at $t=0$ such that the carrier gets back to the initial one for $t>t_{0}$ for some $t_{0} \in \mathbb{Z}_{>0}$, but not for $0 \leq t \leq t_{0}$. In the other words, it is a finite sequence of non-vacuum states which reduces to solitons at $t=t_{0}+1>0$. In the following examples, we have $t_{0}=0$ in (i) and $t_{0}=1$ in (ii).

Example 8.3. Relaxation solitons.

(i) $\Phi(3,1)$ :

$$
\begin{aligned}
&\left(\mathbf{Y}_{i}^{t}\right)_{i} \\
& t=0:(00)(23)(00)(00)(00)(00)(00)(00) \\
& t=1:(00)(11)(30)(00)(00)(00)(00)(00) \\
& t=2:(00)(01)(40)(00)(00)(00)(00)(00) \\
& t=3:(00)(00)(41)(00)(00)(00)(00)(00) \\
& t=4:(00)(00)(31)(10)(00)(00)(00)(00) \\
& t=5:(00)(00)(21)(20)(00)(00)(00)(00) \\
& t=6:(00)(00)(11)(30)(00)(00)(00)(00)
\end{aligned}
$$

$\left(\mathbf{Z}_{i}^{t}\right)_{i}$

$(10)(10)(03)(30)(30)(30)(30)(30)$

$(10)(10)(01)(10)(10)(10)(10)(10)$

$(10)(10)(11)(10)(10)(10)(10)(10)$

$(10)(10)(10)(01)(10)(10)(10)(10)$

$(10)(10)(10)(01)(10)(10)(10)(10)$

$(10)(10)(10)(01)(10)(10)(10)(10)$

$(10)(10)(10)(01)(10)(10)(10)(10)$ 
(ii) $\Phi(4,0)$ :

$$
\begin{aligned}
\quad\left(\mathbf{Y}_{i}^{t}\right)_{i} & \left(\mathbf{Z}_{i}^{t}\right)_{i} \\
t=0:(000)(320)(000)(000)(000)(000) & (011)(011)(023)(023)(023)(023) \\
t=1:(000)(041)(000)(000)(000)(000) & (011)(011)(110)(010)(010)(010) \\
t=2:(000)(031)(100)(000)(000)(000) & (011)(011)(110)(011)(011)(011) \\
t=3:(000)(021)(110)(000)(000)(000) & (011)(011)(110)(011)(011)(011) \\
t=4:(000)(011)(120)(000)(000)(000) & (011)(011)(110)(011)(011)(011) \\
t=5:(000)(001)(130)(000)(000)(000) & (011)(011)(101)(011)(011)(011) \\
t=6:(000)(000)(131)(000)(000)(000) & (011)(011)(011)(111)(011)(011) \\
t=7:(000)(000)(031)(100)(000)(000) & (011)(011)(011)(110)(011)(011)
\end{aligned}
$$

We define a pulsar as a finite sequence of non-vacuum states satisfying

(i) the sequence moves to the right with a constant velocity,

(ii') the final carriers $\mathbf{Z}_{i}^{t}$ for $i \gg 1$ are periodic in $t$.

See the following examples.

Example 8.4. Pulsars:

(i) $\Phi(3,1)$ :

$$
\begin{aligned}
\quad\left(\mathbf{Y}_{i}^{t}\right)_{i} & \left(\mathbf{Z}_{i}^{t}\right)_{i} \\
t=0:(00)(10)(00)(00)(00)(00)(00)(00) & (10)(10)(00)(00)(00)(00)(00)(00) \\
t=1:(00)(01)(00)(00)(00)(00)(00)(00) & (10)(10)(11)(20)(20)(20)(20)(20) \\
t=2:(00)(00)(10)(00)(00)(00)(00)(00) & (10)(10)(10)(00)(00)(00)(00)(00) \\
t=3:(00)(00)(01)(00)(00)(00)(00)(00) & (10)(10)(10)(11)(20)(20)(20)(20) \\
t=4:(00)(00)(00)(10)(00)(00)(00)(00) & (10)(10)(10)(10)(00)(00)(00)(00) \\
t=5:(00)(00)(00)(01)(00)(00)(00)(00) & (10)(10)(10)(10)(11)(20)(20)(20)
\end{aligned}
$$

(ii) $\Phi(4,0)$ :

$$
\begin{aligned}
&\left(\mathbf{Y}_{i}^{t}\right)_{i} \\
& t=0:(000)(110)(000)(000)(000)(000) \\
& t=1:(000)(011)(000)(000)(000)(000) \\
& t=2:(000)(001)(100)(000)(000)(000) \\
& t=3:(000)(000)(110)(000)(000)(000) \\
& t=4:(000)(000)(011)(000)(000)(000) \\
& t=5:(000)(000)(001)(100)(000)(000) \\
& t=6:(000)(000)(000)(110)(000)(000)
\end{aligned}
$$

$$
\begin{aligned}
& \left(\mathbf{Z}_{i}^{t}\right)_{i} \\
& (011)(011)(021)(021)(021)(021) \\
& (011)(011)(110)(010)(010)(010) \\
& (011)(011)(101)(002)(002)(002) \\
& (011)(011)(011)(021)(021)(021) \\
& (011)(011)(011)(110)(010)(010) \\
& (011)(011)(011)(101)(002)(002) \\
& (011)(011)(011)(011)(021)(021)
\end{aligned}
$$

8.3. Phase diagram for $\Phi(3,1)$. We close this section with the phase diagram of solitons and pulsars in the case of $\Phi(3,1)$. We numerically observe that when an initial state includes only one non-vacuum state $(x, y) \in\left(\mathbb{Z}_{\geq 0}\right)^{2}$, it is either a positive soliton, a relaxation soliton, or a pulser as follows:

(1) $(x, 1)$ with $x \geq 1$ : a positive soliton, 
(2) $(0,1)$ or $(1,0)$ : a pulsar,

(3) the other $(x, y)$ : a relaxation soliton which reduces to a positive soliton of the minimal form $(x+y-1,1)$.

If we consider the dynamical system on $\mathbb{Q}$, the situation is more complicated since there are solitons and pulsars whose minimal lengths are more than one. Nevertheless, we numerically find an interesting structure as shown in Figure 13 .

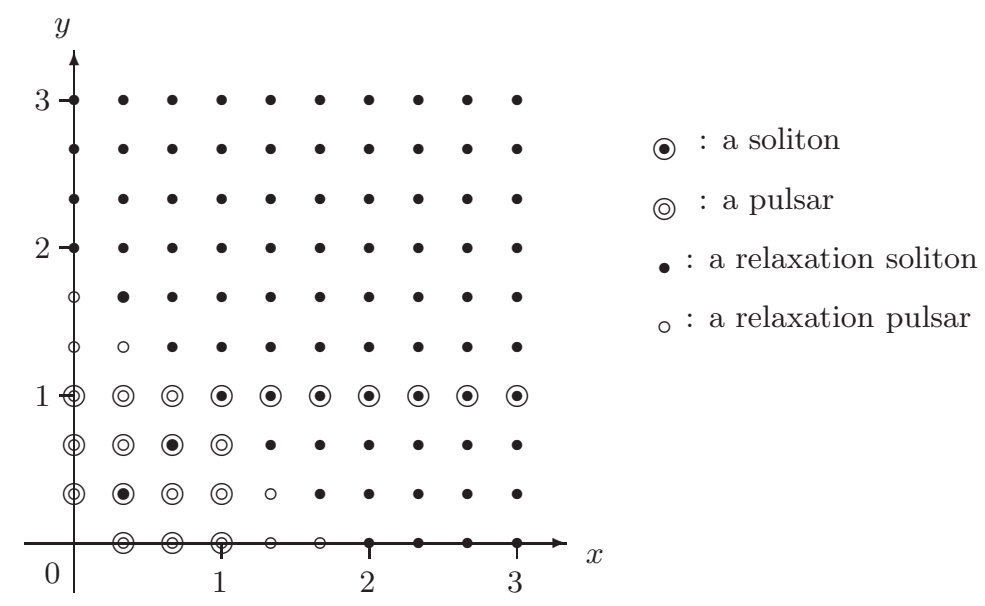

Figure 13. Phase diagram for $\Phi(3,1)$ on $\mathbb{Z} / 3$.

In the phase diagram, the relaxation pulsar is defined to be a finite sequence of nonvacuum states which reduces to a pulsar after a few time steps. The points $(x, y)$ corresponding to solitons are on a line $x=y$ when $x \leq 1$ and on a line $y=1$ when $x \geq 1$. We observe that some relaxation solitons $(x, y)$ are reduced to solitons $(x+y-1,1)$, and the others are reduced to solitons out of the diagram, whose minimal lengths are more than one. This is the same for relaxation pulsars. See Example 8.5 for a relaxation soliton given by $(x, y)=\left(\frac{7}{3}, \frac{2}{3}\right)$, where one sees that the length of the resulting soliton is always two.

Example 8.5. A relaxation soliton of $\Phi(3,1)$ on $\mathbb{Z} / 3$ :

$$
\begin{array}{rlrl} 
& \left(\mathbf{Y}_{i}^{t}\right)_{i} & \left(\mathbf{Z}_{i}^{t}\right)_{i} \\
t & =0:(00)\left(\frac{7}{3} \frac{2}{3}\right)(00)(00)(00)(00)(00)(00) & & (10)(10)\left(0 \frac{2}{3}\right)\left(\frac{2}{3} 0\right)\left(\frac{2}{3} 0\right)\left(\frac{2}{3} 0\right)\left(\frac{2}{3} 0\right)\left(\frac{2}{3} 0\right) \\
t & =1:(00)\left(\frac{4}{3} 1\right)\left(\frac{2}{3} 0\right)(00)(00)(00)(00)(00) & & (10)(10)(01)(10)(10)(10)(10)(10) \\
t & =2:(00)\left(\frac{1}{3} 1\right)\left(\frac{5}{3} 0\right)(00)(00)(00)(00)(00) & & (10)(10)\left(\frac{2}{3} 1\right)(10)(10)(10)(10)(10) \\
t & =3:(00)\left(0 \frac{1}{3}\right)\left(2 \frac{2}{3}\right)(00)(00)(00)(00)(00) & & (10)(10)\left(1 \frac{1}{3}\right)\left(\frac{1}{3} \frac{2}{3}\right)(10)(10)(10)(10) \\
t & =4:(00)(00)\left(\frac{4}{3} 1\right)\left(\frac{2}{3} 0\right)(00)(00)(00)(00) & & (10)(10)(10)(01)(10)(10)(10)(10) \\
t & =5:(00)(00)\left(\frac{1}{3} 1\right)\left(\frac{5}{3} 0\right)(00)(00)(00)(00) & & (10)(10)(10)\left(\frac{2}{3} 1\right)(10)(10)(10)(10)
\end{array}
$$

\section{Appendix A. TRopical SEMIFIELD}

A.1. Tropical limit. To a substruction-free rational map, we associate a piecewiselinear map via a limiting procedure called tropicalization.

The algebra $(\mathbb{R} \cup\{\infty\}, \oplus, \odot)$ is called the min-plus algebra (or the tropical semifield), where an addition $\oplus$ and a multiplication $\odot$ are defined by

$$
a \oplus b:=\min [a, b], \quad a \odot b:=a+b .
$$


Note that $\infty$ corresponds to zero in the algebra: we have $\infty \oplus a=a$ and $\infty \odot a=\infty$ for any $a \in \mathbb{R}$. Moreover we have an inverse of $\odot, a \odot(-a)=0$, but not an inverse of $\oplus$. In the following we also write min and + for $\oplus$ and $\odot$.

The substruction-free algebra $\left(\mathbb{R}_{>0},+, \times\right)$ is formally linked to the min-plus algebra in the following way. We define a map $\log _{\varepsilon}: \mathbb{R}_{>0} \rightarrow \mathbb{R}$ with an infinitesimal parameter $\varepsilon>0$ by

$$
\log _{\varepsilon}: a \mapsto-\varepsilon \log a .
$$

For $a>0$, define $A \in \mathbb{R}$ by $a=e^{-\frac{A}{\varepsilon}}$. Then we have $\log _{\varepsilon}(a)=A$. Moreover, for $a, b>0$ define $A, B \in \mathbb{R}$ by $a=e^{-\frac{A}{\varepsilon}}$ and $b=e^{-\frac{B}{\varepsilon}}$. Then we have

$$
\begin{aligned}
& \log _{\varepsilon}(a+b)=-\varepsilon \log \left(e^{-\frac{A}{\varepsilon}}+e^{-\frac{B}{\varepsilon}}\right) \stackrel{\varepsilon \rightarrow 0}{\mapsto} \min (A, B), \\
& \log _{\varepsilon}(a \times b)=A+B .
\end{aligned}
$$

In summary, tropicalization is a procedure which reduce the algebra $\left(\mathbb{R}_{>0},+, \times\right)$ to the min-plus algebra by the procedure $\lim _{\varepsilon \rightarrow 0} \log _{\varepsilon}$ with the scale transformation as $a=e^{-\frac{A}{\varepsilon}}$.

Via tropicalization, substruction-free rational maps on $\mathbb{R}_{>0}$ formally reduce to piecewiselinear maps on $\mathbb{R}$. We may be able to restrict the resulted piecewise-linear map on $\mathbb{R}$ to that on $\mathbb{Z}$, which is sometimes called the ultradiscretization of the original rational map.

A.2. Valuation field. Let $K=\mathbb{C}\{\{t\}\}:=\cup_{n \geq 1} \mathbb{C}\left(\left(t^{1 / n}\right)\right)$ be the field of Puiseux series over $\mathbb{C}$. The field $K$ is an algebraically closed field with non-trivial valuation, where the valuation map val $: K \rightarrow \mathbb{R} \cup\{\infty\}$ is given by

$$
\text { val }: b_{1} t^{a_{1}}+b_{2} t^{a_{2}}+\cdots \mapsto a_{1} \quad \text { if } b_{1} \neq 0, a_{1}<a_{2}<\cdots \in \mathbb{Z} / n \text { for some } n \geq 1 \text {. }
$$

We recall the axioms for the valuation map on $K$ :

(i) $\operatorname{val}(a)=\infty$ iff $a=0$,

(ii) $\operatorname{val}(a b)=\operatorname{val}(a)+\operatorname{val}(b)$ for any $a, b \in K$,

(iii) $\operatorname{val}(a+b) \geq \min [\operatorname{val}(a), \operatorname{val}(b)]$ for any $a, b \in K$.

As for the last axiom, we have an important lemma:

Lemma A.1. For $a, b \in K$, if $\operatorname{val}(a) \neq \operatorname{val}(b)$, then an equality holds in the above (iii), i.e. $\operatorname{val}(a+b)=\min [\operatorname{val}(a), \operatorname{val}(b)]$.

For the proof, see [MS, Lemma 2.1.1] for example.

The tropicalization can be regarded as the following composition map:

$$
\begin{array}{ccccc}
\mathbb{R}_{>0} & \longrightarrow & K & \stackrel{\mathrm{val}}{\longrightarrow} & \mathbb{R} \cup\{\infty\} \\
a=e^{-\frac{A}{\varepsilon}} & \mapsto & t^{A} & \mapsto & A
\end{array}
$$

We write $\operatorname{trop}(a)=A$ for the image $A$ of $a$ under this map.

\section{REFERENCES}

[BFZ] A. Berenstein, S. Fomin and A. Zelevinsky, Parametrizations of canonical bases and totally positive matrices, Adv. in Math., 122, 49-149 (1996).

[FOY] K. Fukuda, M. Okado and Y. Yamada, Energy functions in box ball systems, Internat. J. Modern Phys. A, 15, no. 9, 1379-1392 (2000).

[GP] M. Glick and P. Pylyavskyy, Discrete solitons in infinite reduced words, arXiv:1606.01213.

[HHIKTT] G. Hatayama, K. Hikami, R. Inoue, A. Kuniba, T. Takagi, and T. Tokihiro, The $A_{M}^{(1)}$ automata related to crystals of symmetric tensors, J. Math. Phys., 42, no. 1, 274-308 (2001).

[H1] R. Hirota, The direct method in soliton theory, Cambridge Tracts in Mathematics, 155. Cambridge University Press, Cambridge, 2004.

[H2] R. Hirota, New solutions to the ultradiscrete soliton equations, Stud. Appl. Math., 122, 361-376 (2009). 
[IKT] R. Inoue, A. Kuniba and T. Takagi, Integrable structure of box-ball systems: crystal, Bethe ansatz, ultradiscretization and tropical geometry, J. Phys. A, 45, 073001 (2012).

[ILP] R. Inoue, T. Lam and P. Pylyavskyy, Toric networks, geometric R-matrices and generalized discrete Toda lattices, Comm. Math. Phys., 347, 799-855 (2016).

[IT] R. Inoue and T. Takenawa, Tropical spectral curves and integrable cellular automata, Int. Math. Res. Not. IMRN, no. 9, rnn019, 27 pp. (2008).

[KMT] M. Kanki, J. Mada and T. Tokihiro, Conserved quantutities and generalized solutions of the ultradiscrete KdV equation, J. Phys. A, 44, 145202, 13 pp (2011).

[LP] T. Lam and P. Pylyavskyy, Crystals and total positivity on orientable surfaces, Selecta Math. (N.S.), 19, no. 1, 173-235 (2013).

[LNQ] M. Lobb, F. Nijhoff and G. Quispel, Lagrangian multiform structure for the lattice KP system, J. Phys. A, 42, 11 pp (2009).

[L] G. Lusztig, Total positivity in reductive groups, in 'Lie theory and geometry', vol. 123, Progr. Math., 531-568 (Birkhäuser Boston, Boston, MA, 1994).

[Mi] T. Miwa, On Hirota's difference equations, Proc. Japan Acad. Ser. A Math. Sci., 58, 9-12 (1982).

[N] F. Nijhoff, Advanced discrete systems and integrability, http://www1.maths.leeds.ac.uk/ frank/math5492/AdvLectures.pdf

[MS] D. Maclagan and B. Sturmfels, Introduction to Tropical Geometry, Graduate Studies in Mathematics, vol.161 (AMS, 2015).

[TS] D. Takahashi and J. Satsuma, A soliton cellular automaton, J. Phys. Soc. Japan, 59, no. 10, 3514-3519 (1990)

[TTMS] T. Tokihiro, D. Takahashi, J. Matsukidaira and J. Satsuma, From soliton equations to integrable cellular automata through a limiting procedure, Phys. Rev. Lett. 76 3247-3250 (1996).

[WNSRG] R. Willox, T. Nakata, J. Satsuma, A. Ramani and B. Grammaticos, Solving the ultradiscrete KdV equation, J. Phys. A, 43, 482003, 7 pp (2010).

[Z] A. Zabrodin, A survey of Hirota's difference equations, arXiv:solv-int/9704001.

[ZFSZ] S. Zhao, W. Feng, S. Shen, and J. Zhang, Generalized Cauchy matrix approach for nonautonomous discrete Kadomtsev-Petviashvili system, arXiv:1409.4594v1.

Max Glick, Department of Mathematics, The Ohio State University, Columbus, OH 43210, USA.

E-mail address: glick.107@osu.edu

Rei Inoue, Department of Mathematics and Informatics, Faculty of Science, Chiba UNIVERSITY, CHIBA 263-8522, JAPAN.

E-mail address: reiiy@math.s.chiba-u.ac.jp

Pavlo Pylyavskyy, School of Mathematics, University of Minnesota, Minneapolis, MN 55414, USA.

E-mail address: ppylyavs@umn.edu 